\title{
Celiac disease and its serological pattern in Saudi Arabia: a systematic review
}

\author{
Mohammad-Ayman A Safi ${ }^{1 *}$ and Hadia M-A Safi ${ }^{2}$ \\ ${ }^{1}$ Associate Professor, Department of Medical Microbiology and Parasitology, King Abdulaziz University, Jeddah, Saudi Arabia \\ ${ }^{2}$ Medical intern, Ibn Sina National College for Medical Studies, Jeddah, Saudi Arabia
}

\begin{abstract}
Aim: To analyze the status of Celiac Disease (CD) and its Serological Pattern in Saudi Arabia (SA).

Methods: Using a retrospective systematic literature review, the status of CD in SA was analyzed from various aspects. The related articles (74) were retrieved by database and journal search. Data were analyzed using the statistical package for social science (SPSS Inc).

Results: The retrieved studies mostly represent multi heterogeneity in terms of journals (42 journal), centers (23 center), mode (12 type), fields ( 21 discipline). Most publications were found in: Saudi Medical Journal (12 articles), King Saud University, Riyadh (21), cross sectional (18), retrospective (18), Case report (18), Diabetes mellitus(T1DM) (8); while down syndrome (DS), short status (SS), skeletal health and CD prevalence had 3, 5, 6 and 7 articles respectively. Female to male ratio was 1.95/1. Histological evaluation was introduced early1980s followed by serology in 1990s. Biopsy showed the highest usage (in 57 studies), endomysial-antibody (EMA) (34), tissue-transglutaminase-antibodies (tTG-Ab) (29), anti-gluten-antibodies (AGA) (18), anti-reticulin- antibodies (ARA) (10). Publications started in 1990 with stable annual number (1-3) until it peaked (10 articles), in 2012, 2016 and 2017.
\end{abstract}

Conclusion: The current study represents the first and only Systematic Review concerning the status of CD in SA and its serological pattern. We found one review evaluating skeletal health in CD in SA and one Meta-analysis concerning polymorphism association with CD, but no meta-analysis were found concerning the relationship between CD and other conditions such as T1DM, SS, DS and CD prevalence in SA, which will be our plan for separate publications.

\section{Introduction}

Celiac disease $(\mathrm{CD})$ was first described in the second century $\mathrm{AD}$ by Aretaeus of Cappadocia [1], who used the Greek word "koeliakos", which means "suffering of the bowels". Only in 1888 AD did Samuel Gee [2] give it the classical clinical description. Its cause was explained by the Dutch Pediatrician "Willem K. Dick" who recognized an association between the consumption of bread and cereals and the relapsing diarrhea [3] and that the health of celiac children dramatically improved when wheat, rye and barley were unavailable during the 2nd World War but relapsed at the end of the war when the consumption of wheat flour started afresh in the Netherlands [4].

At the Interlaken meeting of the European Society of Paediatric Gastroenterology and Nutrition (ESPGAN) society in 1969 [5], three diagnostic criteria for celiac disease were proposed, and were further enunciated at the second symposium on celiac disease in 1974 [6], namely:

* Structurally abnormal jejunal mucosa by taking a gluten-containing diet.

* Clear improvement of villous structure by a gluten-free diet.

* Deterioration of the mucosa during challenge.

In 1971, Seah and coworkers observed binding of antibodies in CD patient sera with rodent liver and kidney sections and identified antireticulin antibodies (ARA) [7]. CD serology started initially in the 1970s and 1980s using anti-gluten antibodies (AGA) immunofluorescece followed by ELISA respectively [8,9]. Chorzelski and colleagues (in
1983) described the production of endomysial antibody (EMA) in patients with CD and dermatitis herpetiformis [10]. AGAs also exist in healthy individuals (up to 10\%) and in nonceliac-enteropathies, thus, diagnostic guidelines have recommended that AGA tests should be abandoned for routine diagnosis [11].

In 1990, the ESPGAN revised criteria for diagnosis of celiac disease [12] considered the finding of the circulating antibodies (AGA-IgA, ARA, and EMA) during diagnosis and their disappearance during a gluten free diet (GFD) as valuable to the diagnosis, and emphasized on tow diagnostic criteria with no need for further confirmation: firstly "on the appearance of flat small intestinal mucosa with the histological features of hyperplastic villous atrophy, while the patient is eating adequate amounts of gluten; and secondly on unequivocal and full clinical remission after withdrawal of gluten from the diet".

In 1989, A simple and flexible approach for diagnosis of celiac disease was suggested by Guandalini S, et al. [13].

*Correspondence to: Mohammad-Ayman A Safi, Associate Professor of Immunology, Department of Medical Microbiology and Parasitology, Faculty of Medicine, King Abdul-Aziz University, PO Box 80205, Jeddah 21589, Kingdom of Saudi Arabia, , Tel: +966 (12) 6400000., Ext 21116, Fax: +966 (12) 6403749, E-mail: aymansafi3@hotmail.com

Key words: Saudi Arabia, Celiac disease, Coeliac disease, tTG-Ab, anti-gluten antibody, endomysial antibody, anti-reticulin antibodies

Received: July 24, 2018; Accepted: August 14, 2018; Published: August 16, 2018 
In 1992, Marsh introduced a grading scheme to classify the morphologic spectrum of the mucosa of untreated CD patients [14]. Then, Marsh-Oberhuber classification [15] modified some of Marsh's parameters. In 1997, tissue transglutaminase tTG2 was identified as CD-specific autoantigen [16], which allowed the development of ELISA-based tTGA tests.

The prevalence of $\mathrm{CD}$, which has been underestimated, is now considered one of the most common genetic disorders in the West with a prevalence of $1 \%-2.67 \%$ [17-19]. The literature regarding CD has expanded significantly in the Middle Eastern (ME) and North African (NA) countries during the last 30 years which are mostly epidemiological [20]. Many studies exist on celiac disease (CD) in Saudi Arabia [21-94], but data from these investigations have not yet been compiled. Here, studies on CD in SA were systematically analyzed, classified, characterized and their serological pattern was discussed to highlight the current status of CD in SA, with the aim that the results of this study will contribute in promotion of national guidelines for organized SD research and management.

\section{Strategy for systematic search and study selection}

As shown in the PRISMA flow-diagram (Figure 1); using "celiac disease in Saudi Arabia", "celiac disease in Saudi children" and "prevalence of celiac disease in Saudi Arabia" as key words, an electronic literature search was conducted via PubMed (US National Library of Medicine, with no specific period), Ovid, EBSCO and scholar Google. The retrieved studies (articles) were designed as twelve groups: three groups (according to the three key words) by PubMed search and three groups by EBSCO, three groups by Ovid and three groups by scholar Google. Additional group of articles were obtained through the library of king Fahd research centre of King Abdulaziz University. In addition, we obtained (with thanks) the publications (two articles and one meeting abstract) related to "celiac disease in Saudi Arabia" directly from the editorial department of two local journals (Saudi Journal of Internal Medicine and Journal of King Abdulaziz University Medical Science). All the retrieved articles were checked for matching (duplication) between groups and intra groups via their titles, author(s) and year of publication. The obtained pooled studies (after matching between groups and intra groups) were checked by reading their titles and abstracts, and the full texts for the possibly relevant publications. Articles that were concerned with celiac disease in Saudi Arabia were included. All forms of studies were included (original studies, reviews, guidelines, surveys, questionnaire, letter to the editor, comments and correction; pediatric, adult, both pediatric and adult). Global reviews with author(s) from Saudi centers were also included. The selected (included) articles were arranged in four groups: references of both adult and pediatric subjects, references of adult subjects, references of pediatric subjects and references of animal models. Data from each article of this classification (as four groups) was recorded using statistical package for social science (SPSS Inc), Version 20. Chicago. The recorded data from each article comprised: author(s), date of publication, journal, date of the study, duration, place of the study, single or multi centres, number of patients, female/ male ratio, type of serology and whether with or without biopsy, type of study (cross sectional, retrospective, prospective, case report, letter to the editor, genetic, HLA, Case-control, meta-analysis, review, pilot prospective, animal experiment, correction to previous publication), pattern of study (general pattern, prevalence, genetic, association (with diabetes mellitus, with autoimmune disease(s), with short stature and association with small bowel (SB) damage), animal model, clinical and/or lab characterization (presentation), corrigendum to previous publication, cardiac assessment, diet (GFD) assessment, skeletal assessment, assessment of knowledge, general assessment, effect of breast feeding on autistic CD and effect of thyroidism.

\section{Strategy for studies' grouping}

Age wise, according to Al-Agha, et al. 2015 [33], puberty is defined with a cut-off level of 10 years in females, and 12 years in males; while the term "children and adolescent" denotes "aged one to 18 years" $[33,39,40]$. Thus, age wise, articles were divided into 3 groups: pediatric ( $<12$ years in male, $<10$ years in female or male with females), adults with adolescents ( $>12$ years) and pediatric with adults (and/or adolescents) (> 1 year (or 1-18 years)).

The reference section comprises the included articles [21-94] but not the excluded articles. The included references were serially arranged in two groups: the PubMed references (with PubMed numbers) followed by the non-PubMed references (without PubMed numbers). The PubMed references were further arranged in two subgroups: the "pediatric with adults (and/or adolescents)" group followed by references of "adult with adolescents", as no recorded pediatric references were found. The nonPubMed references were further arranged in three subgroups: "pediatric with adults (and/or adolescents)" group followed by references of "adult with adolescents" then the "pediatric" subgroup. References in each subgroup were arranged in descending chronological order.

According to PubMed classification, children are individuals aged $0-18$ years, while adults are those above 18 years, other definitions of age-groups existed in which individuals aged 19-21 years have been included as children $[95,96]$ indicating that the age group definitions were not always clear. Here, studies which clearly stated that they are concerned with children (without adolescents) or adults were considered accordingly, otherwise they were considered of both children and adults (and/or adolescents).

\section{Statistical analysis}

Analysis of data was performed using statistical package for social science (SPSS Inc), Version 20. Chicago. The results were illustrated in tabulated form and diagrams showing comparisons and frequencies of variables.

\section{Results}

I. Results of the selection process (inclusion/exclusion) of the pertinent Studies (Figure 1 and Table 1): By PubMed search, using "celiac disease in Saudi Arabia" as a key word, 93 references (articles) were retrieved and designed as general group; while by using "celiac disease in Saudi children" as a key word, a group of 52 references were obtained, of which only 8 references were pediatric while the rest 43 references were found among the general 93 references. By PubMed search, using "prevalence of celiac disease in Saudi Arabia" as a key word, a group of 35 references were obtained, of which only 6 references were not matching with the previous two groups of references.

By Ovid search, the results and the Search Strategy were as follows:

A-Search for Celiac disease in Saudi Arabia (1946-March, weak 3 , 2018). We obtained 20 articles by using the following Search Strategy:

1- Celiac Disease/ ( $\mathrm{N}=18464)$

2- Saudi Arabia/(N=10586)

3- 1 and $2(\mathrm{~N}=20)$; which were used, 


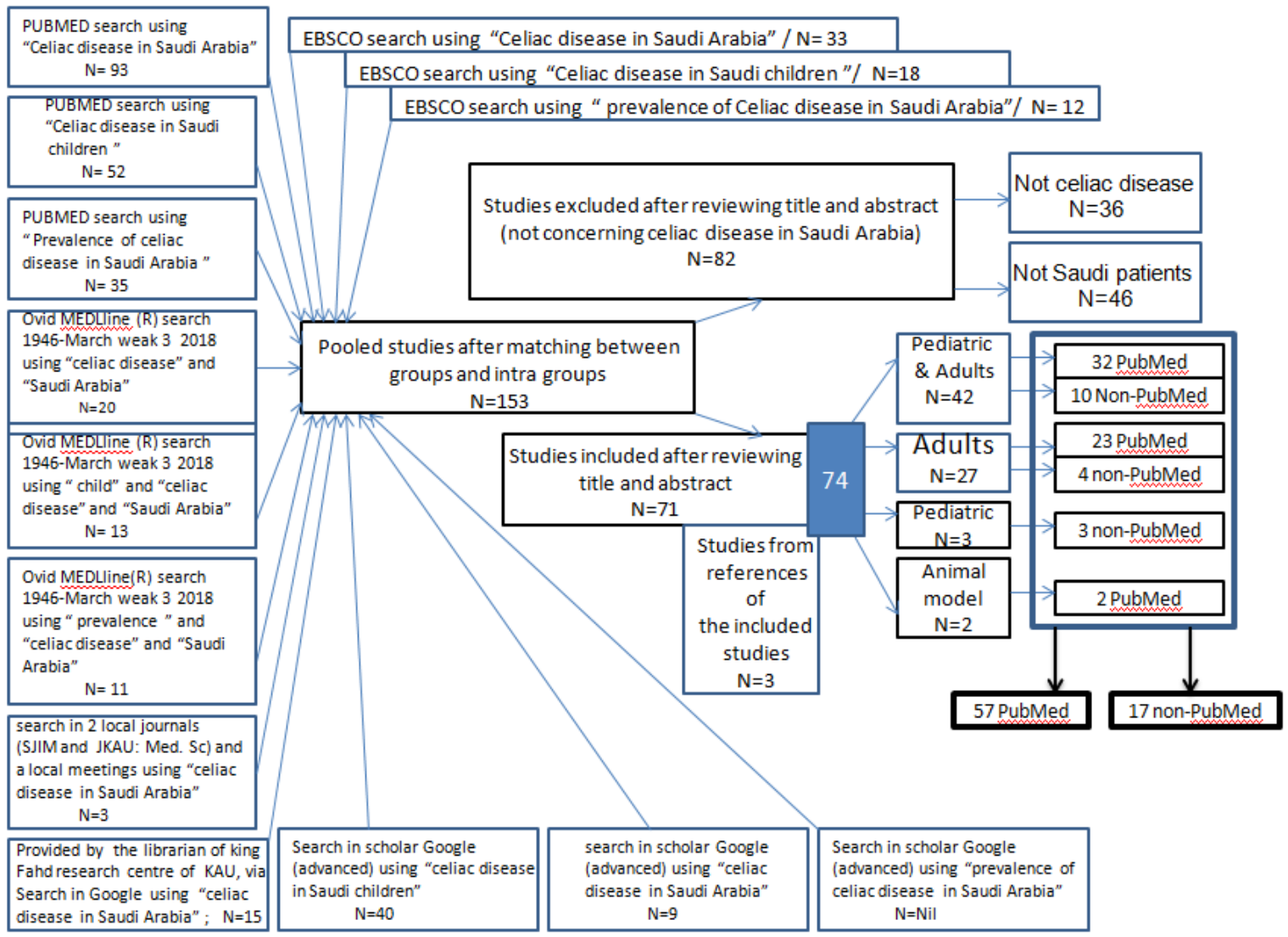

Figure 1. PRISMA flow-diagram showing the selection process (inclusion/exclusion) of the pertinent Studies

Table 1. Age pattern of selected (identified) studies on celiac disease (CD) in Saudi Arabia

\begin{tabular}{|c|c|c|c|c|c|c|c|}
\hline & & & $\begin{array}{l}\text { Pediatric \& Adult } \\
\text { (and/or adolescent) } \\
\text { (0ne year and over) }\end{array}$ & $\begin{array}{c}\text { Adult } \\
\text { (and/or adolescent) } \\
(>12 \text { years })\end{array}$ & $\begin{aligned} & \text { Pediatric } \\
< & 12 \text { years } / \mathrm{M} \\
< & 10 \text { years/F) }\end{aligned}$ & $\begin{array}{c}\text { Animal } \\
\text { model }\end{array}$ & \\
\hline \multirow[t]{5}{*}{ Saudi* } & & Celiac $^{\wedge} \quad$ No & 42 & 27 & 3 & 2 & 74 \\
\hline & & References & $\begin{array}{l}21-52 \\
78-87\end{array}$ & $\begin{array}{l}53-75 \\
88-91\end{array}$ & $92-94$ & $76-77$ & $\begin{array}{l}\text { 21-77 PubMed } \\
\text { 78-94 non-PubMed }\end{array}$ \\
\hline & & Not celiac ${ }^{\wedge \wedge}$ No & & & & & 36 \\
\hline & & References & & & & & excluded \\
\hline & Total & & & & & & 110 \\
\hline \multirow[t]{2}{*}{ non-Saudi** } & Total & No & & & & & 46 \\
\hline & & References & & & & & excluded \\
\hline Total & & (153 pooled + & of the pooled-include & studies) 156 & & & \\
\hline
\end{tabular}

*Saudi $=$ articles concerned with Saudi patients or animal studies in Saudi Arabia .

** non-Saudi= articles concerned with non-Saudi patients or animal studies not in Saudi Arabia.

${ }^{\wedge} \mathrm{celiac}=$ articles concerned with celiac disease.

$\wedge \wedge$ articles concerned with non-celiac disease(s).

B- Search for Celiac disease in Saudi Children (1946-March weak 3 2018). We obtained 13 articles by using the following Search Strategy:

1- Child / ( $=1544508)$

2- Celiac Disease/ ( $\mathrm{N}=18464)$

3- Saudi Arabia/(N=10586)

4- 1 and 2 and $3(\mathrm{~N}=13)$ which were used,
C- Search for Prevalence of celiac disease in Saudi Arabia (1946-March weak 3 2018). We obtained 11 articles by using the following Search Strategy:

1- Prevalence/ $(\mathrm{N}=246629)$

2- Celiac Disease/ ( $\mathrm{N}=18476)$

3- Saudi Arabia/(N=10601)

4- 1 and 2 and $3(\mathrm{~N}=11)$ which were used, 
By EBSCO search using:

- "Celiac disease in Saudi Arabia" (N=33).

- "Celiac disease in Saudi children" ( $\mathrm{N}=18)$.

- "prevalence of Celiac disease in Saudi Arabia" ( $\mathrm{N}=12)$

All the EBSCO articles were also PubMed, that match with the previous PubMed articles.

By search in scholar Google (advanced) using:

- "celiac disease in Saudi children" $(\mathrm{N}=40)$

- "celiac disease in Saudi Arabia" (N=9)

- "prevalence of celiac disease in Saudi Arabia"/ N=Nil

Only eight studies were new non-PubMed articles

Fifteen articles were obtained through the library of king Fahd research centre of King Abdulaziz University. Two articles and one meeting abstract, related to "celiac disease in Saudi Arabia", were obtained directly from the editorial department of two local journals (Saudi Journal of Internal Medicine and Journal of King Abdulaziz University Medical Science).
All the retrieved references (articles) were checked for matching intra and between groups via their titles, author(s) and year of publication. Following the matching process, 153 pooled articles were obtained. 82 articles were excluded that were not concerned with celiac disease in Saudi Arabia; they were 36 not celiac articles and 46 articles not related to Saudi Arabia. Three additional non-PubMed studies were obtained from the references of the included articles: Two pediatric and one adult.

The sum of the included articles were 74 references (see references). The 74 references (articles) [21-94] comprised of two groups: 57 PubMed references [21-77] and 17 non-PubMed references [78-94]. The 57 PubMed references comprised three subgroups: 32 references of "pediatric and adult (and/or adolescents)" [21-52], 23 references of "adult with adolescents" [53-75] and 2 animal references [76,77]. The 17 non-PubMed references comprised of three subgroups: 10 "pediatric with adults (and/or adolescents)" [78-87], 4 "adult with adolescents" [88-91] and three "pediatric" [92-94]. References in each subgroup were arranged (in the reference section) in a descending chronological order.

II. Female to male ratio: Gender information was found in 53 articles, while missing [not specified (NS) or not applicable (NA)] in

Table 2. Classification and characterization of identified studies on celiac disease (CD) in Saudi Arabia, with their serological/biopsypattern

\begin{tabular}{|c|c|c|c|c|c|c|c|c|c|}
\hline $\begin{array}{l}\text { Reference } \\
\text { number }\end{array}$ & journal* & Type** & Author(s)/Date & Prevalence & $\begin{array}{l}\text { F/M Ratio } \\
\text { (Age Y) }\end{array}$ & $\begin{array}{l}\text { Cohort } \\
\text { (Age Year) }\end{array}$ & $\begin{array}{l}\text { Center*** } \\
\text { (Multi-\#) }\end{array}$ & Period & serology/biopsy pattern \\
\hline \multicolumn{10}{|c|}{ Case report $=17$ articles } \\
\hline 35 & 34 & 4 & $\begin{array}{l}\text { Al-Hussaini A, } \\
\text { etal/2013 }\end{array}$ & Not applicable & $\begin{array}{l}1 / 0 \\
(11)\end{array}$ & one patient case study & $12(2)$ & case study & TTG-IgA, and biopsy \\
\hline 49 & 46 & 4 & $\begin{array}{l}\text { Al Hemidan AI, } \\
\text { etal/2006 }\end{array}$ & Not applicable & $\begin{array}{l}1 / 0 \\
(3)\end{array}$ & one patient case study & $13(3)$ & case study & TTG-IgA, EMA-IgA, and biopsy \\
\hline 50 & 43 & 4 & $\begin{array}{l}\text { Al Furaikh S, } \\
\text { etal/2005 }\end{array}$ & Not applicable & $\begin{array}{l}2 / 1 \\
(25,30,42)\end{array}$ & 3patients case study & $16(1)$ & case study & TTG-IgA, and biopsy \\
\hline 55 & 20 & 4 & $\begin{array}{l}\text { Tarabzouni S, } \\
\text { AlKhairallah } \\
\text { T/2017 }\end{array}$ & Not applicable & $\begin{array}{l}1 / 0 \\
(44)\end{array}$ & one case report & $7(1)$ & NS & EMA-IgA, and biopsy \\
\hline 57 & 23 & 4 & Lihabi AA/2016 & Not applicable & $\begin{array}{l}1 / 0 \\
(22)\end{array}$ & $\begin{array}{l}\text { a case of } 22 \text {-year-old } \\
\text { Saudi female }\end{array}$ & $8(1)$ & $\begin{array}{l}\text { Not } \\
\text { applicable }\end{array}$ & $\begin{array}{l}\text { Two years ago, diagnosed with CD } \\
\text { ( No details of the used lab tests) }\end{array}$ \\
\hline 60 & 2 & 4 & $\begin{array}{l}\text { Hasanato RM, } \\
\text { Almomen } \\
\text { AM/2015 }\end{array}$ & Not applicable & $\begin{array}{l}1 / 0 \\
(39)\end{array}$ & one case study & 2(1) & case study & CD patient on GFD \\
\hline 62 & 25 & 4 & $\begin{array}{l}\text { Al-Judaibi B } \\
\text { etal/2015 }\end{array}$ & Not applicable & $\begin{array}{l}1 / 0 \\
(68)\end{array}$ & one case study & $2(2)$ & case study & TTG and biopsy \\
\hline 65 & 38 & 4 & $\begin{array}{l}\text { Masoodi I } \\
\text { etal/2012 }\end{array}$ & Not applicable & $\begin{array}{l}0 / 1 \\
(27)\end{array}$ & one patient case study & $12(1)$ & case study & TTG-IgA, and biopsy \\
\hline 68 & 2. & 4 & $\begin{array}{l}\text { Azzam NA } \\
\text { etal/2006 }\end{array}$ & Not applicable & $\begin{array}{l}1 / 0 \\
(30)\end{array}$ & one patient case study & $13(1)$ & case study & TTG-IgA, EMA-IgA, and biopsy \\
\hline 69 & 47 & 4 & $\begin{array}{l}\text { Al-Gahtani } \\
\text { HA/2005 }\end{array}$ & Not applicable & $\begin{array}{l}2 / 0 \\
(28,32)\end{array}$ & 2 cases & 1(1) & case study & emeil to author \\
\hline 70 & 4 & 4 & $\begin{array}{l}\text { Yasawy MI } \\
\text { etal/2004 }\end{array}$ & Not applicable & $\begin{array}{l}2 / 1 \\
(25,30,42)\end{array}$ & 3 cases & $19(1)$ & case study & EMA, AGA (ARA in 2), and biopsy in 3 \\
\hline 73 & 6 & 4 & $\begin{array}{l}\text { Al-Humaidi } \\
\text { MA/2001 }\end{array}$ & Not applicable & $\begin{array}{l}1 / 0 \\
(22)\end{array}$ & $\begin{array}{l}\text { one patient case } \\
\text { report }\end{array}$ & $20(1)$ & case study & IgG and IgA AGA and biopsy \\
\hline 74 & 2 & 4 & $\begin{array}{l}\text { Ahluwalia M } \\
\text { etal/1996 }\end{array}$ & Not applicable & $\begin{array}{l}1 / 0 \\
(25)\end{array}$ & one patient case study & $21(2)$ & case study & AGA,ARA and biopsy \\
\hline 75 & 15 & 4 & $\begin{array}{l}\text { Nazer HM } \\
\text { etal/1991 }\end{array}$ & Not applicable & $\begin{array}{l}2 / 1 \\
(11,15,4)\end{array}$ & $\begin{array}{l}3 \\
\text { lympangueradeectasia } \\
\text { masq }\end{array}$ & $13(1)$ & case study & biopsy and GFD no improvement \\
\hline 88 & 50 & 4 & Alahdal AM/2017 & Not applicable & $\begin{array}{l}0 / 1 \\
(44)\end{array}$ & one case & 1(1) & case study & TTG-IgA, and biopsy \\
\hline 89 & 53 & 4 & $\begin{array}{l}\text { Ahmed M-E K } \\
\text { etal/2014 }\end{array}$ & Not applicable & $\begin{array}{l}0 / 1 \\
(70)\end{array}$ & one case & $20(1)$ & case study & AGA, EMA-IgA, and biopsy \\
\hline 91 & 6 & 1 & $\begin{array}{l}\text { Zawawi TH et } \\
\text { al/1996 }\end{array}$ & Not applicable & $\begin{array}{l}5 / 1 \\
(19-54)\end{array}$ & 6 & $30(2)$ & 1988-1994 & EMA.AGA, ARA, and biopsy \\
\hline
\end{tabular}




\begin{tabular}{|c|c|c|c|c|c|c|c|c|c|}
\hline \multicolumn{10}{|c|}{ Association with diabetes mellitus $=8$ articles } \\
\hline 27 & 6 & 1 & $\begin{array}{l}\text { Al-Hakami } \\
\text { AM/2016 }\end{array}$ & by serology $\left(21 / 202=10.4^{\circ}\right.$ & \begin{tabular}{ll|} 
(0) & $\begin{array}{l}13 / 8 \\
(1-21)\end{array}$
\end{tabular} & 202 T1DM patients & $20(1)$ & March 2013 to June 2014. & EMA and TTG \\
\hline 33 & 6 & 1 & $\begin{array}{l}\text { Al-Agha AE } \\
\text { etal/2015 }\end{array}$ & $\begin{array}{l}\text { By serology and biopsy } \\
? ? / 228=19.7 \%\end{array}$ & $\begin{array}{l}\text { NS } \\
(1-18)\end{array}$ & 228 children & 1(1) & $\begin{array}{l}\text { January } 2013 \text { to January } \\
2014\end{array}$ & TTG-IgA and biopsy \\
\hline 37 & 35 & 1 & $\begin{array}{l}\text { Al-Hussaini A, } \\
\text { etal/2012 }\end{array}$ & $\begin{array}{l}\text {-By serology }(19 / 106= \\
17.9 \%)- \text { Biopsy proven } \mathrm{CD} \\
12 / 106=(11.3 \%)\end{array}$ & $\begin{array}{l}11 / 1 \\
(8 \mathrm{~m}- \\
15.5)\end{array}$ & 106 children & $12(1)$ & prospective; 208-2010 & $\begin{array}{l}\text { TTG-IgA, EMA-IgA, and } \\
\text { biopsy }\end{array}$ \\
\hline 39 & 6 & 2 & $\begin{array}{l}\text { Saadah OI, } \\
\text { etal } / 2012\end{array}$ & $\begin{array}{l}\text {-By serology }(91 / 430= \\
21.2 \%) \\
- \text { Biopsy proven CD } \\
48 / 430=(11.2 \%)\end{array}$ & \begin{tabular}{l|l} 
NS & 430 \\
$(1.1-18)$ & 430
\end{tabular} & 430 children & 1(1) & $\begin{array}{l}\text { retrospective hospital } \\
\text { record-based study of all } \\
\text { children and adolescents } \\
\text { with T1DM who were } \\
\text { screened for CD }\end{array}$ & TTG-IgA and biopsy \\
\hline 51 & 6 & 1 & $\begin{array}{l}\text { Al-Ashwal AA, } \\
\text { etal/2003 }\end{array}$ & $\begin{array}{l}\text {-By serology }(10 / 123= \\
8.1 \%) \\
\text {-Biopsy proven CD } \\
6 / 123=4.9 \%\end{array}$ & $\begin{array}{l}2 / 8 \\
(2-33)\end{array}$ & $\begin{array}{l}123 \text { patients (10 with } \\
\text { CD) }\end{array}$ & $1(1)$ & between 1995 and 1996 & $\begin{array}{l}\text { AGA,ARA IgA, and } \\
\text { biopsy }\end{array}$ \\
\hline 78 & 56 & 2 & $\begin{array}{l}\text { Alghamdi RA, } \\
\text { etal/2018 }\end{array}$ & $\begin{array}{l}\text { By serology } \\
19 / 268=7.1 \%\end{array}$ & $\begin{array}{l}11 / 8 \\
(2-23)\end{array}$ & $\begin{array}{l}268 \\
(19 \text { with CD) }\end{array}$ & $4(3)$ & NS & TTG \\
\hline 83 & 55 & 1 & $\begin{array}{l}\text { Alshareef MA, } \\
\text { etal } / 2016\end{array}$ & $\begin{array}{l}\text {-By serology }(16 / 218= \\
7.3 \%) \\
- \text { Biopsy proven CD } \\
10 / 218=4.6 \%\end{array}$ & \begin{tabular}{l|l}
$12 / 4$ & 218 \\
$(12-50)$ & $(16$
\end{tabular} & $\begin{array}{l}218 \\
(16 \text { with } \mathrm{CD})\end{array}$ & $28(1)$ & \multirow[t]{2}{*}{$\begin{array}{l}\text { between January } 2008 \text { and } \\
\text { June } 2009\end{array}$} & \begin{tabular}{l|l} 
and & TTG and biopsy
\end{tabular} \\
\hline 87 & 60 & 2 & $\begin{array}{l}\text { Saadah OI } \\
\text {,etal/2004 }\end{array}$ & $\begin{array}{l}\text {-By serology }(23 / 110= \\
21 \%) \\
\text {-Biopsy proven CD } \\
11 / 110=10 \%\end{array}$ & $\begin{array}{l}\text { NS } \\
(1-18)\end{array}$ & $\begin{array}{l}\text { diabetic children } \\
\text { adolescents } \\
8 \mathrm{Y})\end{array}$ & 1(1) & & AGA,TTG,biopsy \\
\hline \multicolumn{10}{|c|}{ Prevalence $=7$ articles } \\
\hline 23 & 7 & 1 & $\begin{array}{l}\text { Al-Hussaini A, } \\
\text { etal/2017 }\end{array}$ & $\begin{array}{l}- \text { By serology }(221 / 7930= \\
2.78 \%) \\
\text {-Biopsy and/or ESPHAN } \\
\text { criteria } \\
(119 / 7930=1.5 \%)\end{array}$ & $\begin{array}{l}81 / 38 \\
(6-15)\end{array}$ & $\begin{array}{l}7930 \\
(6-15 Y)\end{array}$ & 7(3) & $\begin{array}{l}\text { January } 2014 \text { to June } \\
2016\end{array}$ & $\begin{array}{l}\text { TTG-IgA, EMA-IgA, and } \\
\text { biopsy }\end{array}$ \\
\hline 26 & 25 & 2 & $\begin{array}{l}\text { Al-Hakami } \\
\text { AM/2016 }\end{array}$ & $\begin{array}{l}\text { By serology }(58 / 315= \\
18.4 \% \text { of at-risk subjects }) \\
\text {-Biopsy proven CD } \\
(22 / 315=6.9 \%)\end{array}$ & $\begin{array}{l}40 / 18 \\
(1-79 Y)\end{array}$ & $\begin{array}{l}315 \text { at-risk subjects } \\
(176 \mathrm{~F}) \\
(1-79 \mathrm{Y}) \\
(40 \mathrm{had} \\
\text { endoscopy })\end{array}$ & $6(1)$ & $\begin{array}{l}2009 \text { to- } 2012 \\
\text { At- risk subjects }\end{array}$ & $\begin{array}{l}\text { TTG-IgA, EMA-IgA, and } \\
\text { biopsy }\end{array}$ \\
\hline 28 & 4 & 5 & $\begin{array}{l}\text { Al-Mendalawi } \\
\text { MD/2016 }\end{array}$ & Not applicable & $\begin{array}{l}\text { Not applicable } \\
\text { (comment) }\end{array}$ & Not applicable & $9(1)$ & Not applicable & None/comments \\
\hline 29 & 4 & 5 & $\begin{array}{l}\text { Almadi MA, } \\
\text { Aljebreen AM/2016 }\end{array}$ & Not applicable & $\begin{array}{l}\text { Not applicable } \\
\text { (comment) }\end{array}$ & Not applicable & 2(2) & Not applicable & None/comments \\
\hline 30 & 4 & 1 & $\begin{array}{l}\text { Al Hatlani } \\
\text { MM/2015 }\end{array}$ & $\begin{array}{l}3 \% \text { by-tTG } 1 \% \text { by } \\
\text { biopsy }\end{array}$ & $\begin{array}{l}6 / 4 \\
(6-18)\end{array}$ & 1141 participants & $10(1)$ & $\begin{array}{l}\text { Between } 2012 \text { and } \\
2014\end{array}$ & $\begin{array}{l}\text { IgA-TTG) and IgG-TTG. } \\
\text { biopsy was offered to all }\end{array}$ \\
\hline 63 & 1 & 3 & $\begin{array}{l}\text { Aljebreen AM, } \\
\text { etal/2013 }\end{array}$ & $\begin{array}{l}\text { By serology }(26 / 1167=2.2 \\
\%)\end{array}$ & $\begin{array}{l}17 / 9 \\
(16-18)\end{array}$ & 1167 students & 2(3) & $\begin{array}{l}\text { December } 2007 \text { - } \\
\text { January } 2008\end{array}$ & EMA-IgA and EMA-IgG \\
\hline 64 & 4 & 11 & Khayyat YM/2012 & By serology $(3 / 204=1.5 \%)$ & $\begin{array}{l}1 / 2 \\
\text { (mean 35) }\end{array}$ & 204 blood donors & $26(1)$ & NS & TTG-IgA \\
\hline \multicolumn{10}{|c|}{$C D$ and skeletal conditions $=6$ articles } \\
\hline 36 & 2 & 2 & Assiri A, etal/2013 & $\begin{array}{l}10 / 26(38.4 \%) \\
\text { CD in rickets }\end{array}$ & $\begin{array}{l}3 / 2 \\
1-15)\end{array}$ & 26 & 2(1) & 2000 to $2010(10 \mathrm{Y})$ & $\begin{array}{l}\text { TTG-IgA, EMA-IgA, and } \\
\text { biopsy }\end{array}$ \\
\hline 38 & 36 & 10 & $\begin{array}{l}\text { Fouda MA, } \\
\text { eta/2012 }\end{array}$ & Global literature review & $\begin{array}{l}\text { Not applicable } \\
\text { review) }\end{array}$ & Literature review & 2(1) & $\begin{array}{l}\text { Literature review } \\
(1996-2010)\end{array}$ & None/review \\
\hline 42 & 10 & 2 & $\begin{array}{l}\text { A1 Jurayyan NA, } \\
\text { etal/2012 }\end{array}$ & $\begin{array}{l}6.17 \% \text { Rickets du } \\
\text { to } \mathrm{CD}\end{array}$ & $\begin{array}{l}5 / 0 \\
2-18)\end{array}$ & 81 & 2(1) & $\begin{array}{l}\text { january } 1990 \text { to } \\
\text { December } 2009\end{array}$ & EMA,AGa,ARA an biopsy \\
\hline 61 & 6 & 1 & $\begin{array}{l}\text { Assiri AM, } \\
\text { etal/2015 }\end{array}$ & Physicians' survey & $\begin{array}{l}\text { Not applicable } \\
\text { Adults) }\end{array}$ & $\begin{array}{l}109 \text { physicians } \\
\text { completed the } \\
\text { survey }\end{array}$ & 2(3) & $\begin{array}{l}\text { January } 2013 \text { - } \\
\text { January } 2104\end{array}$ & None/survey \\
\hline 84 & 52 & 8 & Jouhari O/2013 & $\begin{array}{l}43 \% \text { of IBD had Low } \\
\text { BMD }\end{array}$ & $\begin{array}{l}8 / 34 \\
3-18)\end{array}$ & $\begin{array}{l}72 \text { IBD }(61 \% \\
\text { Croh's and 39\% } \\
\text { CD) }\end{array}$ & $1(1)$ & NS & $\begin{array}{l}\text { No details of serology or } \\
\text { histology }\end{array}$ \\
\hline 90 & 51 & 3 & $\begin{array}{l}\text { Othman HI and } \\
\text { Ouda SA/2012 }\end{array}$ & $\begin{array}{l}70 \% \text { of } C D \text { had low } \\
\text { BMD }\end{array}$ & $\begin{array}{l}24 / 36 \\
22-46)\end{array}$ & 60 & $1(1)$ & NS & $\begin{array}{l}\text { No details of serology or } \\
\text { histology }\end{array}$ \\
\hline \multicolumn{10}{|c|}{ Genetic $=5$ articles } \\
\hline 31 & 28 & 8 & $\begin{array}{l}\text { Saadah O,I } \\
\text { etal/2015 }\end{array}$ & Not applicable & $\begin{array}{l}1 / 46 \\
57 \text { adolescent } \\
\text { and } 40 \text { child) }\end{array}$ & $\begin{array}{l}97 \text { unrelated } \\
\text { patients and } 123 \\
\text { controls }\end{array}$ & 1(3) & case control study & $\begin{array}{l}\text { AGA,TTG-IgA, EMA-IgA, and } \\
\text { biopsy }\end{array}$ \\
\hline
\end{tabular}




\begin{tabular}{|c|c|c|c|c|c|c|c|c|c|c|c|}
\hline 54 & \multicolumn{2}{|c|}{19} & 8 & $\begin{array}{l}\text { Al-Aama JY, } \\
\text { etal/2017 }\end{array}$ & \multicolumn{2}{|c|}{ Not applicable } & $\begin{array}{l}6 / 45 \\
21-19)\end{array}$ & $\begin{array}{l}100 \text { patients and } \\
100 \text { controls }\end{array}$ & 1(1) & NS & $\begin{array}{l}\text { TTG-IgA, EMA-IgA, and } \\
\text { biopsy }\end{array}$ \\
\hline 56 & \multicolumn{2}{|l|}{21} & 6 & $\begin{array}{l}\text { Banaganapalli B, } \\
\text { etal/2017 }\end{array}$ & \multicolumn{2}{|l|}{ Not applicable } & $\begin{array}{l}\text { IS } \\
\text { P\&Adolscents) }\end{array}$ & $\begin{array}{l}15 \text { patients after } \\
\text { remission and } 15 \\
\text { controls }\end{array}$ & $1(1)$ & NS & CD proven \\
\hline 58 & \multicolumn{2}{|l|}{9} & 9 & Khan S, etal/2016 & \multicolumn{2}{|c|}{ Not applicable } & Iot applicapble & Meta-analysis & $5(3)$ & Not applicable & $\begin{array}{l}\text { meta analysis of } 11 \text { case control } \\
\text { studies } \\
\text { Polymorphism is Associated } \\
\text { with CD }\end{array}$ \\
\hline 59 & \multicolumn{2}{|l|}{49} & 2 & Al-Ajlan AS/2016 & \multicolumn{2}{|c|}{$\begin{array}{l}1.9 \% \text { of controls and } \\
9.6 \% \text { of IBD }\end{array}$} & $\begin{array}{l}2 / 23 \\
20-60)\end{array}$ & $\begin{array}{l}482 \text { controls and } \\
498 \text { IBD }\end{array}$ & 2(1) & $\begin{array}{l}\text { January } 2012 \text { to } \\
\text { January } 2013\end{array}$ & $\begin{array}{l}\text { TTG-IgA, EMA-IgA, and } \\
\text { biopsy }\end{array}$ \\
\hline \multicolumn{12}{|c|}{ Association with short stature $=5$ articles } \\
\hline 41 & \multicolumn{2}{|c|}{10} & 2 & $\begin{array}{l}\text { Al-Jurayyan N NA, } \\
\text { etal/2012 }\end{array}$ & \multicolumn{2}{|c|}{$\begin{array}{l}\text { Biopsy proven } \\
(3 / 110=2.59 \%)\end{array}$} & $\begin{array}{l}\text { NS } \\
(2.5-14)\end{array}$ & 110 & 2(1) & $\begin{array}{l}\text { January } 1990 \text { and } \\
\text { December } 2009\end{array}$ & celiac screening and biopsy \\
\hline 45 & 41 & & 3 & Assiri AM/2010 & \multicolumn{2}{|c|}{$\begin{array}{l}\text {-Seropositive } \\
(15 / 91=16.5 \%) \\
\text { - biopsy proven } 10 / 91 \\
(10.9 \%) \\
\text {-potential CD }(4.3 \%)\end{array}$} & $\begin{array}{l}9 / 6 \\
(4.5-12)\end{array}$ & 91 & 2(1) & $\begin{array}{l}\text { August } 2002 \text { to } \\
\text { December } 2008\end{array}$ & $\begin{array}{l}\text { TTG-IgA, EMA-IgA, and } \\
\text { biopsy }\end{array}$ \\
\hline 47 & \multicolumn{2}{|l|}{40} & 1 & $\begin{array}{l}\text { Al-Ruhaily AD, } \\
\text { Malabu UH/2009. }\end{array}$ & \multicolumn{2}{|c|}{$\begin{array}{l}\text { Serology and biopsy } \\
\text { proven }(4 / 104=4 \%)\end{array}$} & $\begin{array}{l}\text { NS for CD } \\
(12-21)\end{array}$ & 104 subjects & 14(1) & $\begin{array}{l}\text { January } 1997 \text { to } \\
\text { December } 2006 .\end{array}$ & EMA and biopsy \\
\hline 86 & \multicolumn{2}{|c|}{60} & 2 & $\begin{array}{l}\text { Saadah OI, } \\
\text { etal/2004 }\end{array}$ & \multicolumn{2}{|c|}{$\begin{array}{l}24 \%(15 / 63) \\
\text { serologically; } 9.5 \% \text { (6/63) } \\
\text { biopsy proven }\end{array}$} & $\begin{array}{l}\text { Not applicable } \\
(1.37-17.6)\end{array}$ & $\begin{array}{l}63 \text { children and } \\
\text { adolescents with } \\
\text { short stature }\end{array}$ & $1(1)$ & retrospectively & AGA,TTG,biopsy \\
\hline 92 & \multicolumn{2}{|c|}{58} & 1 & $\begin{array}{l}\text { Al-Jurayyan NAM, } \\
\text { etal/2013 }\end{array}$ & \multicolumn{2}{|c|}{$\begin{array}{l}\text { Biopsy proven } \\
(5 / 110=4.5 \%)\end{array}$} & $\begin{array}{l}\text { NS } \\
(2.5-14)\end{array}$ & 110 & $8(2)$ & $\begin{array}{l}\text { January } 1990 \text { - } \\
\text { December } 2009\end{array}$ & EMA and biopsy \\
\hline \multicolumn{12}{|c|}{ Clinical and/or lab characterization (presentation) $=5$ articles } \\
\hline 21 & \multicolumn{2}{|l|}{4} & 1 & Fouda MA/2017. & \multicolumn{2}{|l|}{$86.9 \%$} & $\begin{array}{l}149 / 69 \\
(1-75)\end{array}$ & $\begin{array}{l}218(90 \\
\text { children and } \\
128 \text { adults })\end{array}$ & 2(1) & 2003 and 2012. & $\begin{array}{l}\text { TTG-IgA, EMA-IgA, and } \\
\text { biopsy }\end{array}$ \\
\hline 22 & 6 & & 2 & Saeed A, etal/2017 & $20.1 \%$ of biop & osies & $\begin{array}{l}30 / 29 \\
(1-18)\end{array}$ & $64 / 318(20.1 \%$ & 2(1) & $\begin{array}{l}\text { January } 2009 \text { to } \\
\text { January } 2015\end{array}$ & TTG-IgA, and biopsy \\
\hline 71 & 2 & & 8 & Al Attas RA/2002 & $\begin{array}{l}\text { By biopsy : } 4 . \\
\text { By EMA: } 7.6^{\circ}\end{array}$ & $\begin{array}{l}.0 \% \text { in } \mathrm{CD} \\
\% \text { in } \mathrm{CD}\end{array}$ & $\begin{array}{l}64 / 81 \\
(0.9-36)\end{array}$ & $\begin{array}{l}145 \mathrm{CD}, \\
100 \text { healthy } \\
\text { blood donors } \\
\text { controls }\end{array}$ & $15(1)$ & prospective & EMA and ARA \\
\hline 72 & 6 & & 2. & Qari FA/2002 & Not applicable & & $\begin{array}{l}14 / 2 \\
\text { (mean37.5) }\end{array}$ & 16 & $1(1)$ & 5 years from $1998-2$ & $\begin{array}{l}002 \text { Biopsy and improvment by } \\
\text { GFD }\end{array}$ \\
\hline 93 & 6 & & 2 & $\begin{array}{l}\text { Abdullah AMA } \\
/ 1990\end{array}$ & Not applicable & & $\begin{array}{l}\text { NS } \\
(20-60)\end{array}$ & 19 & 2(1) & retrospectively & $\begin{array}{l}\text { Biopsy and improvement } \\
\text { by GFD }\end{array}$ \\
\hline & ion $\mathbf{w}$ & h au & oimn & e disease $(s)=3$ article & & & & & & & \\
\hline 44 & 6 & 2 & & bas BS/2010 & $\begin{array}{l}1 / 20(\mathrm{CD} \text { in } \\
\text { APS-1) }\end{array}$ & $\begin{array}{l}1 / 0 \\
(16)\end{array}$ & $\begin{array}{l}20 \text { (APS-1) } \\
1 \text { Fem/with CD }\end{array}$ & 13(1) & January & 00 to December & AGA,ARA,EMA,TTGA,biopsy \\
\hline 52 & 1 & 3 & & youf SM/2011 & $\begin{array}{l}42.8 \%(18 / 42) \\
\text { Sero+ in JRA }\end{array}$ & $\begin{array}{l}10 / 8 \\
(5-15)\end{array}$ & $\begin{array}{l}42 \text { Juvenile } \\
\text { RA(JRA) }\end{array}$ & $13(1)$ & NS & & $\begin{array}{l}\text { AGA-IgA,AGA-IgG, ARA and } \\
\text { EMA-IgA } \\
\text { and biopsy }\end{array}$ \\
\hline 76 & 19 & 12 & & e $\mathrm{A} / 2014$ & Not applicable & $\begin{array}{l}\text { Not applicable } \\
\text { (Mouse model) }\end{array}$ & mouse model & $11(3)$ & $\begin{array}{l}\text { anti-idio } \\
\text { related } t\end{array}$ & $\begin{array}{l}\text { pe Ab structure } \\
\mathrm{D} \text { (mouse model) }\end{array}$ & animal model \\
\hline & ion $\mathbf{w}$ & h Do & wn sy & rome $=3$ articles & & & & & & & \\
\hline 40 & 6 & 2 & & OI, etal $/ 2012$ & $\begin{array}{l}2 / 51=4 \% \text { by ttg } \\
\text { and } 1 / 51=2 \% \\
\text { by biopsy }\end{array}$ & $\begin{array}{l}0 / 1 \\
(0.5-16.6)\end{array}$ & 51 & 1(1) & January & 07 - August 2011 & TTG-IgA and biopsy \\
\hline 82 & 59 & 2 & & raily $F$, etal/2017 & $\begin{array}{l}27 / 84 \\
=32.14 \% \\
\text { AGA-IgA+ve } \\
9 / 84=10.9 \% \\
\text { biopsy } \\
\text { confirmed } \\
10.7 \%\end{array}$ & $\begin{array}{l}\text { Not } \\
\text { applicapble } \\
(1-18)\end{array}$ & 84 & $13(1)$ & retrospe & vely & EMA,TTG,AGA,biopsy \\
\hline
\end{tabular}




\begin{tabular}{|c|c|c|c|c|c|c|c|c|c|c|}
\hline 85 & 45 & 2 & AL Mehaidib, et al/2011 & $\begin{array}{l}45 / 91 \\
=49.45 \% \\
\text { seropositive } \\
9 / 91=9.8 \% \\
\text { by biopsy } \\
\text { confirmed }\end{array}$ & 8/1(1.3-17.6) & 91 & 13(1) & \multicolumn{2}{|l|}{ retrospectively } & Ema,TTG,AGA,ARA,Biopsy \\
\hline \multicolumn{11}{|c|}{ Pattern of celiac disease in adolescent and/or children $=3$ articles } \\
\hline 43 & 2 & 2 & Saadah OI/2011 & Not applicable & $44 / 36(0.5-18)$ & 80 & 1(1) & \multicolumn{2}{|c|}{ September 2002 -July 2007} & TTG and biopsy \\
\hline 48 & 43 & 1 & Assiri AM, etal/2008 & Not applicable & $\begin{array}{l}\text { NS in abstract } \\
(0.3 \mathrm{~m}-14 \mathrm{Y})\end{array}$ & 62/first year & 2(1) & \multicolumn{2}{|c|}{ retrospectively 10 years } & $\begin{array}{l}\text { EMA. AGA ARA, and } \\
\text { biopsy }\end{array}$ \\
\hline 94 & 2 & 2 & Nazer HM, etal/1990 & Not applicable & NS( 0.9-16y) & 22 & 13(1) & \multicolumn{2}{|l|}{$1987-1988$} & $\begin{array}{l}\text { Biopsy and improvement } \\
\text { by GFD }\end{array}$ \\
\hline \multicolumn{11}{|c|}{ Diet $=\mathbf{2}$ articles } \\
\hline 32 & 24. & 1.00 & Sarkhy AA etal/2015 & Not applicable & $71 / 42(\geq 18)$ & 113 & 2(3) & \multicolumn{2}{|c|}{ November 2013- January 2014} & children with biopsy \\
\hline 25 & 24. & 1.00 & Sarkhy A eta/2016 & Not applicable & $71 / 42(\geq 18)$ & 113 & 2(3) & \multicolumn{2}{|l|}{ Not applicable } & children with biopsy \\
\hline \multicolumn{11}{|c|}{ Corrigendum to previous publication $=1$ article } \\
\hline 53 & 7 & 1 & Fouda MA, etal/2017 & $1.5 \%$ & $\begin{array}{l}\text { Not applicable } \\
\text { (correction) }\end{array}$ & $\begin{array}{l}\text { Not applicable } \\
\text { (correction) }\end{array}$ & $7(1)$ & \multicolumn{2}{|c|}{$\begin{array}{l}\text { Not applicable (correction to } \\
\text { reference 38) }\end{array}$} & None/correction \\
\hline \multicolumn{11}{|c|}{ Aassociation with cardiac conditions $=1$ article } \\
\hline 24 & 4 & 8 & Fathy A, etal/2016 & Not applicable & $\begin{array}{l}12 / 8 \\
(8.2 \pm 2.7)\end{array}$ & \begin{tabular}{|l}
20 CD children and \\
20 healthy controls
\end{tabular} & $8(1)$ & \multicolumn{2}{|c|}{$\begin{array}{l}\text { Prospective; February and march } \\
2015\end{array}$} & $\begin{array}{l}\text { TTG-IgA, EMA-IgA, and } \\
\text { biopsy }\end{array}$ \\
\hline \multicolumn{11}{|c|}{ General review $=1$ article } \\
\hline 34 & 33 & 10 & $\begin{array}{l}\text { Guandalini S1, Assiri } \\
\text { A/2014 }\end{array}$ & Not applicable & \begin{tabular}{|l}
\multicolumn{1}{c}{ Not } \\
applicable \\
(review)
\end{tabular} & review & 2(1) & \multicolumn{2}{|l|}{ review } & review \\
\hline \multicolumn{11}{|c|}{ Breast feeding and $\mathrm{CD}$ and autistic $=1$ article } \\
\hline 77 & 1 & 12 & $\begin{array}{l}\text { Selim ME, Al-Ayadhi } \\
\text { LY/2013 }\end{array}$ & Not applicable & $\begin{array}{l}\text { Not } \\
\text { applicable } \\
\text { (Female rats) }\end{array}$ & animal model & 2(1) & \multicolumn{2}{|l|}{ animal model } & animal model20 \\
\hline \multicolumn{11}{|c|}{ Effect of thyroidism $=1$ article } \\
\hline 67 & 44 & 4 & $\begin{array}{l}\text { Alzahrani AS, Al Sheef } \\
\text { M/2008 }\end{array}$ & Not applicable & $1 / 0(24)$ & $\begin{array}{l}\text { one patient case } \\
\text { study }\end{array}$ & 13(1) & \multicolumn{2}{|l|}{ case study } & $\begin{array}{l}\text { CD proven by serological } \\
\text { screen and biopsy }\end{array}$ \\
\hline \multicolumn{11}{|c|}{ CD and pregnancy $=1$ article } \\
\hline 66 & 12 & 4 & Jammah AA/2012 & Not applicable & $1 / 0(25)$ & $\begin{array}{l}\text { one patient case } \\
\text { study }\end{array}$ & 2(1) & case study & \multicolumn{2}{|c|}{ TTG-IgA, EMA-IgA, and biopsy } \\
\hline \multicolumn{11}{|c|}{ Genetic and dental $=1$ article } \\
\hline 46 & 42 & 8 & Ouda S, etal $/ 2010$ & Not applicable & $\begin{array}{l}32 / 18 \\
(10-18)\end{array}$ & $\begin{array}{l}50 \mathrm{CD} \text { and } 50 \\
\text { controls }\end{array}$ & 1(1) & prospective & \multicolumn{2}{|c|}{ CD proven by biopsy } \\
\hline \multicolumn{11}{|c|}{ Psycatric $=1$ article } \\
\hline 81 & 54 & 1 & Alharbi KL, etal/2017 & \begin{tabular}{|l}
$43.1 \%$ \\
depression
\end{tabular} & $\begin{array}{l}91 / 18 \\
(<15->45)\end{array}$ & 109 & $27(1)$ & $\begin{array}{l}\text { June and July } \\
2017\end{array}$ & \multicolumn{2}{|c|}{ CD proven by serological screen and biopsy } \\
\hline \multicolumn{11}{|c|}{ Extraabdominal conditions pattern $=1$ article } \\
\hline 80 & 57 & 1 & Albrady ö, etal/2018 & $\begin{array}{l}14.8 \% \text { have } \\
\text { osteoporosis }\end{array}$ & \begin{tabular}{|l}
$502 /$ \\
167 \\
$(18-50)$
\end{tabular} & 669 responses. & $27(3)$ & $\begin{array}{l}28 \text { February } \\
2017 \text { until } 8 \\
\text { July } 2017\end{array}$ & \multicolumn{2}{|c|}{ online questionnaire. } \\
\hline \multicolumn{11}{|c|}{ Quality of life in CD 29=1 article } \\
\hline 79 & 57 & 1 & Al-Qefari SA, etal/2018 & Not applicable & 192/72(5-64) & 264 & $27(1)$ & NS & \multicolumn{2}{|c|}{ survey (SF-36) questionnaire } \\
\hline
\end{tabular}

*Journals(Total 42): 1=World J Gastroenterol, 2=Ann Saudi Med, 4=Saudi J Gastroenterol, 6= Saudi Med J, 9=J Pediatr Gastroenterol Nutr, 10=Sci Rep,12=Sudan J Paediatr, 15=Osteoporos Int,16=Ann Trop Paediat,19= Can J Gastroenterol Hepatol, 20=PLoS One, 21=J Mov Disord, 23=J Cell Biochem, 24=Case Rep Gastroentero, 25=Pediatr Gastroenterol Hepatol Nutr, 28=Arab J Gastroenterol, 33=Dis Markers, 34=JAMA Pediat, 35=Ann Hepatol, 36=BMC Gastroenterol, 38=Can J Gastroenterol, 40=Eur J Gastroenterol Hepatol, 41=Niger J Med, 42=Pediatr Rep, 43=J Clin Pediatr Dent, 44=Trop Gastroenterol, 45=Endocr Pract, 46=J Gastroenterol Hepatol, 47=Eur J Ophthalmol, 48=Neurosciences (Riyadh), 49=J Biol Sci, 50=Saudi J Intern Med, 51=JKAU: Med. Sci, 52=,9th Applied Medical Sciences Students' Annual Meeting; February 19, 2013; King bdulaziz University, 53=American Journal of Medicine and Medical Sciences, 54=Annals of Medical and Health Sciences Research, 54=Int J Diabetes Metab Disord, 55=IOSR Journal of Pharmacy and Biological Sciences (IOSR-JPBS), 56=Ann Med Health Sci Res, 57=Journal of Taibah University Medical Sciences, 59=International Journal of Pediatrics and Adolescent Medicine, 60=Journal of Pediatric Gastroenterology and Nutrition.

** Type of study: $1=$ Cross sectional, $2=$ Retrospective, $3=$ Prospective, $4=$ Case report, $5=$ Letter to the editor, $6=$ Genetic, $8=$ Case-control, $9=$ Meta analysis, $10=$ Review, $11=$ Pilot prospective, $12=$ animal experiment, $13=$ Corrigendum to previous publication.

***Centers: 1=KAU-jedda; $2=$ King Saud University, Riyadh; $3=$ not Saudi; $4=29=$ King Fahd Hospital, Al-Baha, Saudi Arabia; $5=$ University of Ha'il; $6=$ King Khalid University; $7=$ Alfaisal University, Riyadh; 8= Taibah University, Madinah; 9= Baghdad University; 10= King Abdullah International Medical Research Center-Riyadh; 11= King Abdullah University of Science and Technology, Thuwal; 12= King Fahad Medical City, Riyadh; 13= King Faisal Specialist Hospital and Research Center-Riyadh; 14= The Townsville Hospital, Australia; 15= Regional Laboratory and Blood Bank, Ministry of Health, Dammam; 16=King Fahad National Guard Hospital, Saudi Arabia.; 17=Abdul-Aziz Medical City, Jeddah; 18=Good Hope Hospital NHS - Sutton - UK; 19= King Fahad Hospital of the University, AI-Khobar, Saudi Arabia; 20= King Khalid University, Abha; 21= Al Mana General Hospital, Al Khobar; 22= Queen Rania Alabdullah Hospital of Pediatrics, King Hussein Center, Amman, Jordan; 23= Alkadra Teaching Hospital, Tripoli, Libya; 24= Alkadra Teaching Hospital, Tripoli, Libya; $25=$ Shaheed Beheshti University, Tehran, Iran;26Umm AlQura University; $27=$ Qassim University; 28= King Fahad Armed Forces Hospital, Jeddah; 30= King Khalid National Guard Hospital,Jeddah.

\#Multi : 1=author(s) is (are) from one center; $2=$ authors are from two centers; $3=$ authors are from muli-ceners 
Table 3. Female to male pattern

\begin{tabular}{|l|c|c|c|c|c|c|c|}
\hline & $\begin{array}{c}\text { Number } \\
\text { of Studies }\end{array}$ & $\begin{array}{c}\text { Minimum } \\
\text { count }\end{array}$ & $\begin{array}{c}\text { Maximum } \\
\text { count }\end{array}$ & Sum & Mean & $\begin{array}{c}\text { Std. } \\
\text { Deviation }\end{array}$ & $\begin{array}{c}\text { Female } \\
\text { /male ratio }\end{array}$ \\
\hline Female & 53 & 0 & 502 & 1731 & 33.88 & 75.88 & \multirow{1}{*}{$1731 / 884=1.95$} \\
\hline Male & 53 & 0 & 167 & 884 & 17.31 & 29.50 & \\
\hline
\end{tabular}

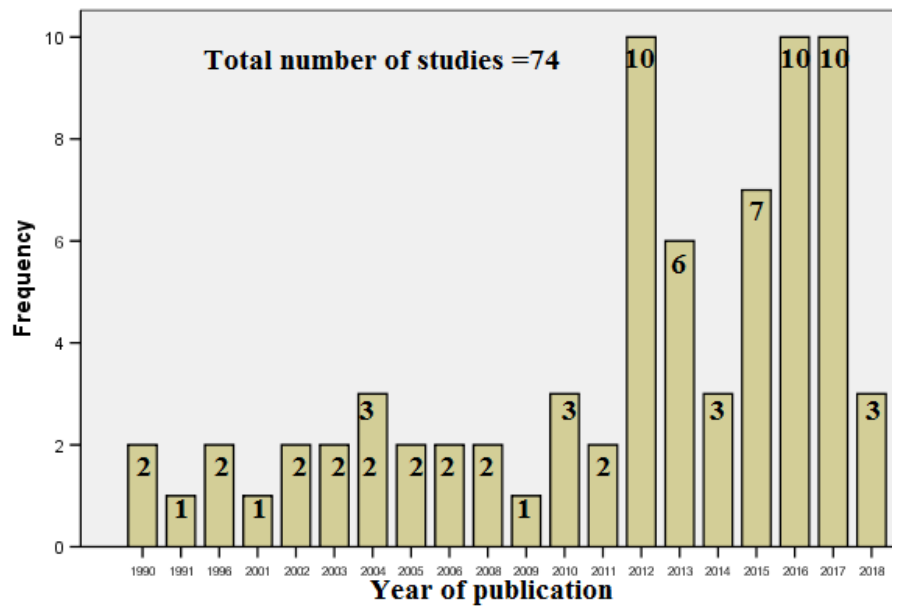

Figure 2. Distribution of studies (Total 74) according to the year of publication

21 articles (Table 2). Articles with "female more than male" were five time (45/8) those with "male more than female" (Table 2). The ratio of the total female (1731) over the total male (884) was 1.95 /1 (Table 3).

III. Duration span: Publications concerning CD in SA started in 1990 (Figure 2) with stable annual number (1-3) until it peaked (10 articles) in 2012, 2016 and 2017. The two earliest studies of $1990[93,94]$ covered the retrospective period from 1978.

IV. Cohort: In this study, cohort is meant to be the number of human subjects that were involved in each study whether patients of CD (for different evaluations) or of other conditions (for CD association), normal subjects (for CD prevalence), normal controls (in the case control studies) and participants in surveys.

Information about cohort were found in 66 articles, while missing [not specified (NS) or not applicable (NA)] in 8 articles (2 animal model, 2 comments, 1 correction, 2 reviews, 1 meta-analysis) (Table 2). The 66 articles with cohorts' information included: 14 articles of one case studies [35,49,57,55,60,62,65,67,66,68,73,74,88,89], one article of two case studies [69], three articles of three case studies [50,70,75] and 48 articles [21-34, 36-48, 51-54,56,58,59,61,63,64, 71,72, 76-87,90-94] with a range of 6 - 7930 subjects (Table 2).

V. Journals: The selected studies were published in 42 journals. Saudi Med J showed the highest (31 publications), followed by Saudi J Gastroenterol and Ann Saudi Med (7 publications each), World J Gastroenterol (3 publications), then Seven journals with two publications each and finally, thirty-one journals with one publication each (Figure 3).

VI. Centers: Studies were related to 23 centers representing almost the entire kingdom of SA. King Saud University, Riyadh, showed the highest number of studies (21), followed by KAU-Jeddah (14), King Faisal Specialist Hospital and Research Center-Riyadh (10), Alfaisal University, Riyadh (2), four centers with 3 studies each, and fourteen centers (with one study each) which included two centers from outside the kingdom: the Townsville Hospital [29], and Baghdad University [28] concerning comments on another article from SA (Figure 4).
VII. Types versus fields (disciplines) of studies: Twelve types were found. Most publications were found as cross sectional, retrospective and case report (18 each). Followed by case-control 6, prospective 4, review 2 , letter to the editor 2 , animal experiment 2 , genetic 1 , Metaanalysis 1 , Pilot prospective 1 , corrigendum to previous publication 1 .

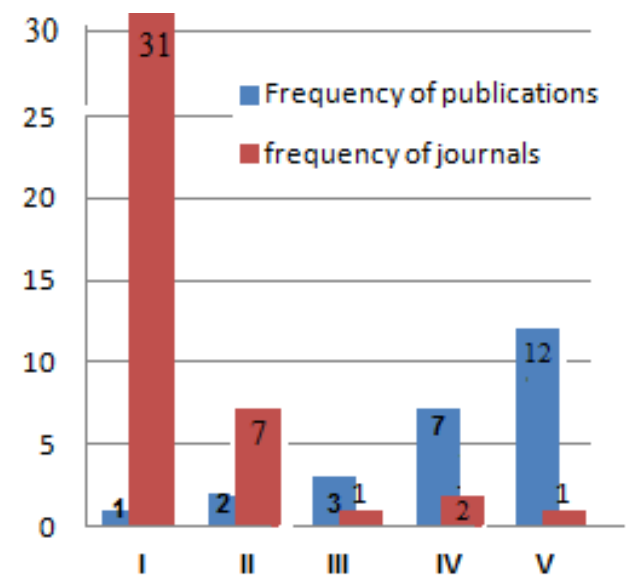

Figure 3. Frequency of journals (Total 42) versus frequency of publications (Total 74) I=Thirty one journals wth one publication each: J Pediatr Gastroenterol Nutr, Sci Rep,Osteoporos Int, Ann Trop Paediat, Can J Gastroenterol Hepatol, J Mov Disord, J Cell Biochem, Case Rep Gastroentero, Dis Markers, JAMA Pediatrm, Ann Hepatol, BMC Gastroenterol, Can J Gastroenterol, Eur J Gastroenterol Hepatol, Niger J Med, Pediatr Rep, J Clin Pediatr Dent, Endocr Pract, J Gastroenterol Hepatol, Eur J Ophthalmol, Neurosciences (Riyadh), J Biol Sci, Saudi J Intern Med, JKAU: Med. Sci, 9th Applied Medical Sciences Students' Annual Meeting; February 19, 2013; King bdulaziz University, American Journal of Medicine and Medical Sciences, Annals of Medical and Health Sciences Research, Int J Diabetes Metab Disord, IOSR Journal of Pharmacy and Biological Sciences (IOSR-JPBS), Journal of Taibah University Medical Sciences, International Journal of Pediatrics and Adolescent Medicine,

II=Seven journals wth two publications each: PLoS One, Pediatr Gastroenterol Hepatol Nutr, Arab J Gastroenterol, Trop Gastroenterol, Sudan J Paediatr, Journal of Pediatric Gastroenterology and Nutrition, Ann Med Health Sci Res.

$\mathrm{III}=$ One journal with three publications: World J Gastroenterol

IV=Two journals with seven publications each: Ann Saudi Med and Saudi J Gastroenterol $\mathrm{V}=$ One journal with twelve publications: Saudi Med J

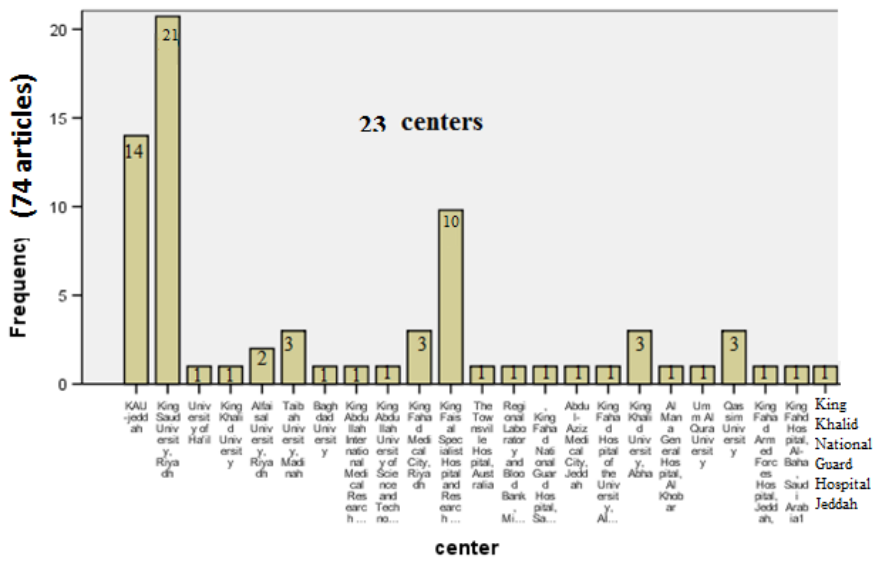

Figure 4. Distribution of articles (Total 74) according to centres (Total 23)

- Fourteen centers with one study each

- One center with two studies

- Four centers with three studies each

- One center with ten studies

- One center with fourteen studies

- One center with twenty-one studies

- Descriptive Statistics 
Table 4. Type of study versus Field of study

\begin{tabular}{|c|c|c|c|c|c|c|c|c|c|c|c|c|c|}
\hline \multirow[b]{2}{*}{ Field } & \multicolumn{11}{|c|}{ Type of study } & \multirow[b]{2}{*}{$\begin{array}{l}\text { Corrigendum } \\
\text { to previous } \\
\text { publication }\end{array}$} & \multirow{2}{*}{ Total (\%) } \\
\hline & $\begin{array}{l}\text { cross } \\
\text { sectional }\end{array}$ & retrospective & prospective & $\begin{array}{l}\text { Case } \\
\text { report }\end{array}$ & $\begin{array}{l}\text { Letter to } \\
\text { the editor }\end{array}$ & genetic & $\begin{array}{l}\text { Case- } \\
\text { control }\end{array}$ & $\begin{array}{l}\text { Meta- } \\
\text { analysis }\end{array}$ & Review & $\begin{array}{l}\text { Pilot } \\
\text { prospective }\end{array}$ & $\begin{array}{l}\text { animal } \\
\text { experiment }\end{array}$ & & \\
\hline prevalence & $\begin{array}{l}2(28.56) \\
{[11.11]}\end{array}$ & $\begin{array}{l}1(14.28) \\
{[5.55]}\end{array}$ & $\begin{array}{l}1(14.28) \\
{[25]}\end{array}$ & & $\begin{array}{l}2(28.56) \\
{[100]}\end{array}$ & & & & & $\begin{array}{l}1(14.28) \\
{[100]}\end{array}$ & & & $7(100.00)\{9.45\}$ \\
\hline Genetic & & $\begin{array}{l}1(20.00 \\
{[5.55]}\end{array}$ & & & & $\begin{array}{l}1(20.00) \\
{[100]}\end{array}$ & $\begin{array}{l}2(40.00) \\
{[33.33]}\end{array}$ & $\begin{array}{l}1(20.00) \\
{[100]}\end{array}$ & & & & & $5(100.00)\{6.75\}$ \\
\hline $\begin{array}{l}\text { association with diabetes } \\
\text { mellitus }\end{array}$ & $\begin{array}{l}5(6.94) \\
{[27.75]}\end{array}$ & $\begin{array}{l}3(4.22) \\
{[15.65\}}\end{array}$ & & & & & & & & & & & $8(100.00)\{10.8\}$ \\
\hline $\begin{array}{l}\text { association with } \\
\text { autoimmune disease(s)) }\end{array}$ & & $\begin{array}{l}1(33,33) \\
{[5.55]}\end{array}$ & $\begin{array}{l}1(33.33) \\
{[25]}\end{array}$ & & & & & & & & $\begin{array}{l}1(33.33) \\
{[50]}\end{array}$ & & $3(100.00)\{4.05\}$ \\
\hline $\begin{array}{l}\text { association with short } \\
\text { stature }\end{array}$ & $\begin{array}{l}2(40.00) \\
{[11.11]}\end{array}$ & $\begin{array}{l}2(40.00) \\
{[11.11]}\end{array}$ & $\begin{array}{l}1(20.00) \\
{[25]}\end{array}$ & & & & & & & & & & $5(100.00)\{6.75\}$ \\
\hline $\begin{array}{l}\text { clinical and/or lab } \\
\text { characterization } \\
\text { (presentation) }\end{array}$ & $\begin{array}{l}1(25.00) \\
{[5.55]}\end{array}$ & $\begin{array}{l}3(50.00) \\
{[15.65]}\end{array}$ & & & & & $\begin{array}{l}1(25.00) \\
{[16.66]}\end{array}$ & & & & & & $5(100.00)\{6.75\}$ \\
\hline case report & $\begin{array}{l}1(5.89) \\
{[5.55]}\end{array}$ & & & $\begin{array}{l}\text { 16(94.11) } \\
{[88.88]}\end{array}$ & & & & & & & & & $\begin{array}{l}17(100.00) \\
\{22.95\}\end{array}$ \\
\hline $\begin{array}{l}\text { Corrigendum to previous } \\
\text { publication }\end{array}$ & & & & & & & & & & & & $\begin{array}{l}1(100.00) \\
{[100]}\end{array}$ & $1(100.00)\{1.35\}$ \\
\hline association with cardiac & & & & & & & $\begin{array}{l}1(100.00) \\
{[16.66]}\end{array}$ & & & & & & $1(100.00)\{1.35\}$ \\
\hline Diet & $\begin{array}{l}2(100.00) \\
{[11.11]}\end{array}$ & & & & & & & & & & & & $2(100.00)\{2.7\}$ \\
\hline $\mathrm{CD}$ and skeletal & \begin{tabular}{|l|}
$1(16.66)$ \\
{$[5.55]$}
\end{tabular} & $\begin{array}{l}2(33.36) \\
{[11.11]}\end{array}$ & $\begin{array}{l}1(16.66) \\
{[25]}\end{array}$ & & & & $\begin{array}{l}1(16.66) \\
{[16.66]}\end{array}$ & & $\begin{array}{l}1(16.66) \\
{[50]}\end{array}$ & & & & $6(100.00)\{8.1\}$ \\
\hline General & & & & & & & & & $\begin{array}{l}1(100.00) \\
{[50]}\end{array}$ & & & & $1(100.00)\{1.35\})$ \\
\hline $\begin{array}{l}\text { Breast fed and CD and } \\
\text { autistic }\end{array}$ & & & & & & & & & & & \begin{tabular}{|l|}
$(100.00)$ \\
{$[50]$}
\end{tabular} & & $1(100.00)\{1.35\}$ \\
\hline $\begin{array}{l}\text { prevalence in Down } \\
\text { syndrome }\end{array}$ & & $\begin{array}{l}3(100.00) \\
{[15.65]}\end{array}$ & & & & & & & & & & & $3(100.00)\{4.05\}$ \\
\hline $\begin{array}{l}\text { Pattern of CD } \\
\text { (1 questionnaire) }\end{array}$ & $\begin{array}{l}1(33.33) \\
{[5.55]}\end{array}$ & $\begin{array}{l}2(66.66) \\
{[11.11]}\end{array}$ & & & & & & & & & & & $3(100.00)\{4.05\}$ \\
\hline effect of thyroidism & & & & $\begin{array}{l}1(100.00) \\
{[5.56]}\end{array}$ & & & & & & & & & $1(100.00)\{1.35\}$ \\
\hline $\mathrm{CD}$ and pregnancy & & & & $\begin{array}{l}1(100.00) \\
{[5.56]}\end{array}$ & & & & & & & & & $1(100.00)\{1.35\}$ \\
\hline genetic and dental & & & & & & & $\begin{array}{l}1(100.00) \\
{[16.66]}\end{array}$ & & & & & & $1(100.00)\{1.35\}$ \\
\hline Psycatric & $\begin{array}{l}1(100.00) \\
{[5.55]}\end{array}$ & & & & & & & & & & & & $1(100.00)\{1.35\}$ \\
\hline $\begin{array}{l}\text { Extraabdominal } \\
\text { conditions pattern }\end{array}$ & $\begin{array}{l}1(100.00) \\
{[5.55]}\end{array}$ & & & & & & & & & & & & $1(100.00)\{1.35\}$ \\
\hline $\begin{array}{l}\text { Quality of life } \\
\text { questionnaire }\end{array}$ & $\begin{array}{l}1(100.00) \\
{[5.55]}\end{array}$ & & & & & & & & & & & & $1(100.00)\{1.35\}$ \\
\hline Total & $\begin{array}{l}18(24.3) \\
{[100]}\end{array}$ & $\begin{array}{l}18(24.3) \\
{[100]}\end{array}$ & $4(5.4)[100]$ & $\begin{array}{l}18(24.3) \\
{[100]}\end{array}$ & $\begin{array}{l}2(2.7) \\
{[100]}\end{array}$ & $\begin{array}{l}1(1.35) \\
{[100]}\end{array}$ & $6(8.1)[100]$ & $\begin{array}{l}1(1.35) \\
{[100]}\end{array}$ & $\begin{array}{l}2(2.7) \\
{[100]}\end{array}$ & $\begin{array}{l}1(1.35) \\
{[100]}\end{array}$ & $\begin{array}{l}2(2.7) \\
{[100]}\end{array}$ & $\begin{array}{l}1(1.35) \\
{[100]}\end{array}$ & $74(100.00)$ \\
\hline
\end{tabular}

The 74 studies involved 21 disciplines: case report (in 17 articles), association with diabetes mellitus (8), prevalence (7), CD and skeletal (6), genetic (5), association with short stature (5), clinical and/or lab characterization (presentation) (5), association with autoimmune disease(s) (3), prevalence in Down syndrome (3), pattern of CD (1 questionnaire) (3), Diet (2). One article for each of the following ten disciplines: general, Breast fed and CD and autistic, association with cardiac, corrigendum to previous publication, effect of thyroidism, $\mathrm{CD}$ and pregnancy, genetic and dental, psychiatric, Extra-abdominal conditions pattern, quality of life (questionnaire).

Distribution of types versus fields (disciplines) and articles are shown in Table $2 \& 4$.

\section{IIX. Results of histology and serology}

\section{- Commencing date}

Histological evaluation was introduced early in1980s [93,94] followed by serology in 1990s for EMA, ARA and AGA [91] and in 2004 for tTG-Ab $[86,87]$ (Table 5).
- $\quad$ Frequency of usage whether as single or combined.

Biopsy showed the highest usage (in 57 studies), tissuetransglutaminase- antibodies (tTG-Ab) (34), endomysial-antibody (EMA) (29), anti-gluten-antibodies (AGA) (18), anti-reticulinantibodies (ARA) (11) (Table 6).

Mode of double combination between different serological tests: tTG-Ab with EMA (in 18 studies), AGA with EMA (12), AGA with ARA (10), ARA with EMA (8), tTG-Ab with AGA (8), tTG-Ab with ARA (2) (Table 7).

Mode of combination between histology and serology in each article: Different patterns of cooperation between biopsy and serology in each article are shown in Table 8 . The concerned information was found in 63 articles, while missing in 11 articles ( 2 animal models, 4 review and questionnaire, 2 comments, one survey, one correction and one with no details). Biopsy was used in 57 studies according to the following pattern of serological cooperation: eleven articles [22,30,3 $3,35,39,40,50,62,65,83,88]$ of tTG-IgA and biopsy; eleven articles [21, 
Table 5. Commencing date of different serology and histology

\begin{tabular}{|c|c|c|c|c|c|}
\hline & AGA & ARA & EMA & TTG & Histology \\
\hline Year & 1996 & 1996 & 1996 & 2004 & 1990 \\
\hline
\end{tabular}

Table 6. Frequency of different serological tests whether as single or combined

\begin{tabular}{|c|c|c|c|c|c|c|}
\hline & & tTG & EMA & AGA & ARA & Biopsy \\
\hline \multirow[t]{9}{*}{ Valid } & Yes & 34 & 29 & 18 & 11 & 53 \\
\hline & No & 19 & 24 & 35 & 42 & 12 \\
\hline & CD proven & 10 & 10 & 10 & 10 & 3 \\
\hline & $\begin{array}{l}\text { biopsy and } \\
\text { improvement by } \\
\text { GFD }\end{array}$ & 3 & 3 & 3 & 3 & \\
\hline & $\begin{array}{l}\text { biopsy and no } \\
\text { improvement by } \\
\text { GFD }\end{array}$ & 1 & 1 & 1 & 1 & \\
\hline & animal model & 2 & 2 & 2 & 2 & 2 \\
\hline & no details & 1 & 1 & 1 & 1 & 1 \\
\hline & $\begin{array}{l}\text { Review and } \\
\text { questionnaire }\end{array}$ & 4 & 4 & 4 & 4 & 4 \\
\hline & Total & 74 & 74 & 74 & 74 & 74 \\
\hline
\end{tabular}

Table 7. Cross-tabulation between different serological tests

\begin{tabular}{|c|c|c|c|c|c|c|c|c|c|c|}
\hline \multirow{2}{*}{ Test } & & \multicolumn{3}{|c|}{ AGA } & \multicolumn{3}{|c|}{ ARA } & \multicolumn{3}{|c|}{ EMA } \\
\hline & & Yes & NO & Total & Yes & NO & Total & Yes & NO & Total \\
\hline \multirow{3}{*}{ tTG } & yes & 8 & 26 & 34 & 2 & 32 & 34 & 18 & 16 & 34 \\
\hline & no & 10 & 9 & 19 & 8 & 11 & 19 & 11 & 8 & 19 \\
\hline & Total & 18 & 35 & 53 & 10 & 43 & 53 & 29 & 24 & 53 \\
\hline \multirow{3}{*}{ AGA } & yes & & & & 10 & 8 & 18 & 12 & 6 & 18 \\
\hline & no & & & & 1 & 34 & 35 & 17 & 18 & 35 \\
\hline & Total & & & & 11 & 42 & 53 & 29 & 24 & 53 \\
\hline \multirow{3}{*}{ ARA } & yes & 10 & 1 & 11 & & & & 8 & 2 & 10 \\
\hline & no & 8 & 34 & 42 & & & & 21 & 22 & 43 \\
\hline & Total & 18 & 35 & 53 & & & & 29 & 24 & 53 \\
\hline
\end{tabular}

23,24,26,36,37,45,49,59,66,68] of tTG-IgA, EMA-IgA and biopsy; ten articles $[25,32,41,46,56,57,58,67,81,90]$ of CD proven by biopsy; five articles [42,48,52,70,91] of AGA,ARA,EMA and biopsy; three articles $[31,54,82]$ of EMA, tTG Ab,AGA and biopsy; three articles $[47,55,92]$ of EMA and Biopsy, three articles $[43,86,87]$ of AGA, tTG Ab and biopsy; three articles $[72,93,94]$ of Biopsy and improvement by GFD; two article $[44,85]$ of EMA, tTG Ab, AGA, ARA and Biopsy; two articles [69,73] of AGA and biopsy; one article [89] of AGA, EMA-IgA, and biopsy; two articles [51,74] of AGA,ARA and biopsy; one article [75] of AGA, EMA-IgA, and biopsy; one article [89] of biopsy and no improvement by GFD. Biopsy was not used in 7 studies according to the following pattern of serological cooperation: two articles [64,78] of tTG-Ab, one article [43] of EMA, one article [71] of EMA and ARA, one article [27] of EMA and tTG Ab, one article [51] of AGA and ARA, one article [60] of EMA, tTG Ab, AGA, ARA. The maximum usage (54\%) was for tTG$\mathrm{Ab}$ (30 with biopsy and 4 without) either alone or in combination with other serological markers; followed by $16 \%$ for CD proven by biopsy, $20 \%$ for markers without tTG-Ab [EMA,ARA,AGA with different combinations] (10 with biopsy and 3 without), $6 \%$ for Biopsy and GFD (3 improved with GFD and 1 no improvement); the minimum usage (4\%) was for AGA-Ab without tTG-Ab (2 with biopsy and EMA or ARA, one with ARA, but never alone). (Table 8)

Chronological usage of serology and biopsy: The pattern of the chronological usage of serology and biopsy in SA is shown in Table 9 and Figure 5. The two earliest studies of $1990[93,94]$ indicated that CD proven by histology (with improvement by GFD) was the mode of diagnosis in 1980s. The study of Zawawi TH, et al. [91], that covered the period 1988-1994, indicated that all serology except tTG-Ab (EMA, AGA and ARA) were used during that period (1989-1994). Also, there was no mention for tTG-Ab in the publications of 2001,2002 and 2003 $[73,71$ and 51 respectively]. The first mention of $\mathrm{tTG}-\mathrm{Ab}$ was found in $2004[86,87]$ indicating its definite presence in 2004.

\section{Discussion}

The current study is a statistical evaluation of all relevant literature on CD in SA, published up to March 2018 with no retrospective date limit. It represents the first and only Systematic Review concerning the

\begin{tabular}{|c|c|c|c|}
\hline \multicolumn{2}{|c|}{ Serology with/without histology } & \multirow{2}{*}{\begin{tabular}{|l|} 
Frequency \\
11
\end{tabular}} & \multirow{2}{*}{$\begin{array}{l}\text { Article's (reference) No } \\
22,30,33,35,39,40,50,62,65 \\
, 83,88\end{array}$} \\
\hline \multirow{13}{*}{$\begin{array}{l}\text { With } \\
\text { biopsy=57 }\end{array}$} & tTG-IgA, and biopsy & & \\
\hline & $\begin{array}{l}\text { tTG-IgA, EMA-IgA, and } \\
\text { biopsy }\end{array}$ & 11 & $\begin{array}{l}21,23,24,26,36,37,45,49,59 \\
, 66,68\end{array}$ \\
\hline & CD proven by biopsy & 10 & $25,32,41,46,56,57,58,67,81,90$ \\
\hline & AGA,ARA,EMA,biopsy & 5 & $42,48,52,70,91$ \\
\hline & $\begin{array}{l}\text { EMA, tTG Ab,AGA and } \\
\text { biopsy }\end{array}$ & 3 & $31,54,82$ \\
\hline & EMA and Biopsy & 3 & $47,55,92$ \\
\hline & AGA, tTG Ab and biopsy & 3 & $43,86,87$ \\
\hline & $\begin{array}{l}\text { Biopsy and improvement } \\
\text { by GFD }\end{array}$ & 3 & $72,93,94$ \\
\hline & $\begin{array}{l}\text { EMA, tTG Ab, AGA, } \\
\text { ARA,Biopsy }\end{array}$ & 2 & 44,85 \\
\hline & AGA and biopsy & 2 & 69,73 \\
\hline & AGA, EMA-IgA, and biopsy & 1 & 89 \\
\hline & AGA,ARA and biopsy & 2 & 74,51 \\
\hline & $\begin{array}{l}\text { Biopsy and no improvement } \\
\text { by GFD }\end{array}$ & 1 & 75 \\
\hline \multirow{5}{*}{$\begin{array}{l}\text { Without } \\
\text { biopsy=6 }\end{array}$} & tTG Ab & 2 & 64,78 \\
\hline & EMA & 1 & 43 \\
\hline & EMA and ARA & 1 & 71 \\
\hline & EMA and tTG Ab & 1 & 27 \\
\hline & EMA, tTG Ab,AGA,ARA & 1 & 60 \\
\hline \multirow{5}{*}{ Missing=11 } & Non & 4 & $28,29,53,61$ \\
\hline & Animal model & 2 & 76,77 \\
\hline & Review and questionnaire & 4 & $34,38.79,80$ \\
\hline & No details & 1 & 84 \\
\hline & Total & 74 & \\
\hline
\end{tabular}

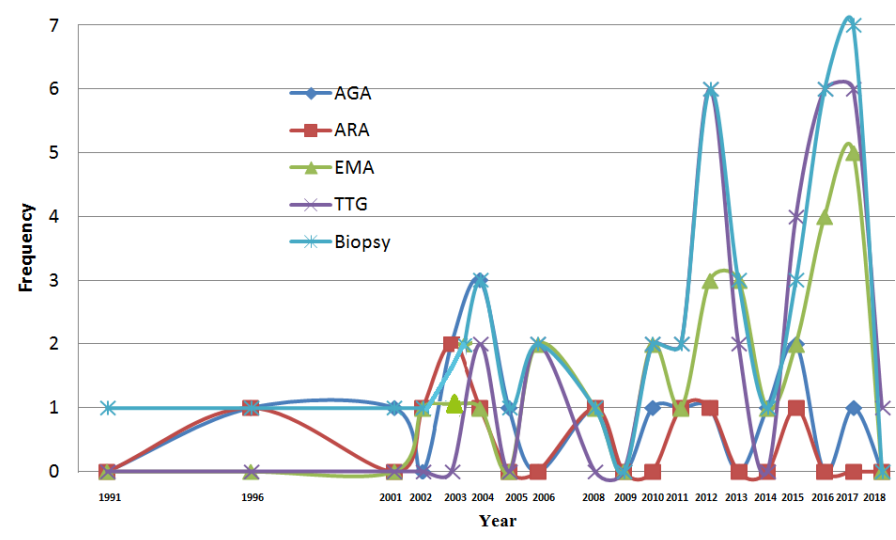

Figure 5. Chronological Pattern of Serology \& Biopsy 
Table 9. Chronological usage pattern of serology and biopsy

\begin{tabular}{|c|c|c|c|c|c|c|c|c|c|c|c|c|c|c|c|c|c|c|c|c|c|}
\hline Year & 1990 & 1991 & 1996 & 2001 & 2002 & 2003 & 2004 & 2005 & 2006 & 2008 & 2009 & 20010 & 2011 & 2012 & 2013 & 2014 & 2015 & 2015 & 2017 & 2018 & Total \\
\hline EMA & 0 & 0 & 1 & 0 & 1 & 1 & 1 & 0 & 2 & 1 & 1 & 2 & 1 & 3 & 3 & 1 & 2 & 4 & 5 & 0 & 29 \\
\hline tTG-Ab & 0 & 0 & 0 & 0 & 0 & 0 & 2 & 1 & 2 & 0 & 0 & 2 & 2 & 6 & 2 & 0 & 4 & 6 & 6 & 1 & 34 \\
\hline AGA & 0 & 0 & 2 & 1 & 0 & 2 & 3 & 1 & 0 & 1 & 0 & 1 & 1 & 1 & 0 & 1 & 2 & 0 & 2 & 0 & 18 \\
\hline ARA & 0 & 0 & 2 & 0 & 1 & 2 & 1 & 0 & 0 & 1 & 0 & 0 & 1 & 1 & 0 & 0 & 1 & 0 & 0 & 0 & 10 \\
\hline Histology & 2 & 1 & 2 & 1 & 1 & 2 & 3 & 2 & 2 & 2 & 1 & 3 & 2 & 7 & 3 & 1 & 4 & 7 & 8 & 0 & 54 \\
\hline
\end{tabular}

status of CD (and its serological pattern) in SA. However, we only found two studies from SA, analyzing the literature concerning specific fields of CD: one Meta-analysis of 11 global case-control studies concerning the genetic polymorphism association with CD [58], and one review (by literature electronic search using the MEDLINE and EMBASE databases) evaluating the global skeletal health (bone mineral density (BMD) and fracture risk) in CD from 1996 to 2010 [38], in addition to a general review about global CD, authored from SA [34].

In the 74 included articles, the ratio of the total female to total male was almost the double (1.95/1). Globally, CD is more common in female (double or triple) than in male, with a possible attribution to the higher frequency of the necessary HLADQ $2 / 8$ alleles in female than male $[97,98]$.

We found different rates of $\mathrm{CD}$ in different studies in SA. $\mathrm{CD}$ prevalence in SA is ranging from $1.5 \%$ to $3 \%$ in four studies $[23,30,63,64]$. The global prevalence is $1 \%$ (with a range of $0.5-1.6 \%$ ) [99-102], with a high prevalence (5.6\%) among the" Saharawi refugees of Berber-Arabic origin" in North Africa [103]. One of the articles [26] studied the seroprevalence of celiac disease in at-risk subjects (T1DM, T1DM + Hypothyroidism, Hypothyroidism, Short stature, Failure to thrive, Abdominal pain, Chronic diarrhea, Anaemia, Others) which was $18.4 \%$. Concerning the global at-risk groups (including Down and Turner syndromes, type 1 diabetes, and autoimmune thyroid disease) the prevalence is ranging from $5 \%$ to $10 \%$ [104].

Concerning the association of $\mathrm{CD}$ with other conditions, we found strongly variable rates of $\mathrm{CD}$ in different conditions in different studies. The prevalence of $\mathrm{CD}$ cases in patients with Rickets differed from $6.87 \%$ to $38.4 \%$ [ 42,36 respectively], while in patients with Low BMD differed from $43 \%$ to $70 \%$ [ 84,90 respectively]. Globally, low bone mineral density (BMD) at both the spine and hip in celiac patients was also reported [105-107]. It was also reported that celiac disease may cause rickets $[108,109]$; even, congenital rickets may be caused by untreated maternal CD [110]. In India, rickets rate within patients suffering of celiac and Type1 Diabetes Mellitus was reported to be $20.8 \%$ [111].

For CD with Diabetes Mellitus (T1DM) [27, 33,37,39,51,78,83,87], down syndrome (DS) $[40,82,85]$ and short status (SS) $[41,45,47,86,92]$; the prevalence ranges were $7.3 \%-19.7 \%, 2-10.7 \%$ and $4 \%-19 \%$ respectively. The Global rates are $4-15 \%$ for Type 1 diabetes mellitus and $5-10 \%$ for Down's syndrome $[112,113]$. In Europe, the CD prevalence among children with short stature is approximately $0.05-056 \%$ [45], while it is $4.0 \%$ in western New York [114].

One article described a case of CD among 20 cases (5\%) of APS type 1 [44], and one article described an association (42.8\%) of CD and juvenile RA [52]. Cases of APS type 2 which were associated with celiac disease were reported $[115,116]$. In Italy, prevalence of CD in RA was nil [117]. In UK, the overall prevalence of celiac disease in RA is $0.63 \%$ [118], while an arthritis is present in $26 \%$ of CD patients, with difference between patients on a regular diet $(41 \%)$ and patients on a gluten-free diet (21.6\%) [119].

Two studies of questionnaire survey to the families of $\mathrm{CD}$ patients. One showed a significant negative socio-economic impact of Gluten-
Free Diet (GFD) on children with CD \& their families [25]. And one questionnaire about the adherence to GFD [32] showed that $60 \%$ of the involved children were reported to be strictly adherent to GFD; the younger age at diagnosis and the shorter duration since the diagnosis the better adherence rate.

Adherence to Gluten-Free Diet can be evaluated by different methods: serology [tTG-Ab (TG2) test], rebiopsy and dietitian interviews or/and questionnaire; however, antibodies take 1.5-3 months to normalize, but the finally, complete histological resolution may not occur until 2 years of GFD [120]. "Celiac Dietary Adherence Test" (CDAT), a 7-question survey that allows a standardized evaluation of adherence and is superior to the tTG-Ab test [121]. Patient interview for monitoring dietary compliance has been proven to be more sensitive than serology [122].

Globally, the degree of strict adherence to a GFD is strongly variable with a range of $42 \%$ to $91 \%$ [123]. Less adherence is among adolescents, and adults diagnosed in childhood; due to lack of knowledge, poor availability and/or labeling of gluten free products, and difficulties in obtaining and/or identifying gluten-free food when dining out [124].

The information, within the articles, indicate for the usage of four serological tests (AGA, ARA, EMA and tTG-Abs), in addition to the biopsy test. Globally, these investigations were described in the fall of the second half of the last century [7-16] (see the introduction). However, with the start of this century (in 2004) a new test (DGPAs) was introduced for the detection of deamidated gliadin peptide Abs, which is promising according to its sensitivity and specificity [125] and appears to be possibly more useful than $\mathrm{tTG}$ in children below the age of 7 years and/or in IgA-immunocompromised patients [126,127]. We found no mention (within the articles) to this new serological test. Recently (in 2013), the first electrochemical immunosensor for DGPAs was described, as a good alternative to the traditional ELISA kits [128].

Amongst the retrieved 74 articles, information of cooperation between biopsy and different serological markers in each article were found within 63 articles, the maximum usage (54\%) was for tTG-Ab, followed by $16 \%$ for $\mathrm{CD}$ proven by biopsy, $20 \%$ for markers without tTG-Ab [EMA, ARA, AGA with different combinations, 6\% for Biopsy and GFD and the minimum usage (4\%) was for AGA-Ab without tTG-Ab. (more detailed description is found in the result section). This type of pattern can be attributed to two reasons: 1- AGA test has low (sensitivity) and \{specificity\} [IgA-AGA (85\%), \{90\%\}; IgGAGA (85\%), \{80\%\}] while tTG-Ab possesses high sensitivity and specificity [IgA-tTG $(98 \%),\{98 \%\}]$, as well as EMA $(95 \%),\{99 \%\}$ and ARA $(72 \%),\{99 \%\}[129,130] .2-$ Tests for ARA and EMA are based on indirect immunofluorescence which requires an individual reading of each sample under a fluorescent microscope, is costly, time consuming and leads to inter-observer variability [131].

HLA typing is also missing in the retrieve articles. This test identifies whether a patient possesses the haplotypes DQ2 or DQ8, the absence of which excludes the possibility of CD [132]. HLA-DQ2 and/or HLA-DQ8 typing is useful in both and diagnosis and epidemiological 
evaluation. Among the global population, HLA-DQ2 and/or HLADQ8 typing is present in $98.6 \%$ of patients with $\mathrm{CD}$, and in $40 \%$ of the general population who do not have the diagnosis of $\mathrm{CD}$ [133].

\section{Conclusion}

Over the last decades, the availability of both specific and sensitive serological markers for $\mathrm{CD}$, accompanied with technical advances have led to improvement in the frequency and accuracy of data on CD in SA. Since 1990, there have been multiple studies concerning the status of CD from many parts of SA, using many types of studies and covering a wide range of conditions, for both the general population and at-risk patients. Most results showed strongly variable rates of $\mathrm{CD}$, among the general population and among at-risk patients, in different studies in SA. However, some conditions still need to be evaluated, such as the small intestinal bacterial overgrowth (SIBO) among patients with celiac disease unresponsive to gluten-free diet (GFD [134,135].

IgA- anti-tTG and AGA showed the highest and the lowest utilization rate respectively, due to their level of sensitivity and specificity which were high [sensitivity (98\%) and specificity $\{98 \%\}$ in tTTG-Ab, in comparison with AGA [(85\%), \{90\%\}]. Although EMA and ARA possess high sensitivity and specificity, they have some drawbacks including low reproducibility and high inter-observer variability, as both based on indirect immunofluorescence which requires an individual reading of each sample under a fluorescent microscope. The literature from SA missed the new currently used test for deamidated gliadin antibodies (DGP-IgG and DGP-IgA), and the DQ2 or DQ8 typing which is employed for both epidemiology and diagnosis, only when negative, to rule out the possibility of CD diagnosis.

\section{Recommendations}

1. Meta-analysis for the different studies of CD prevalence in general population and in each of at-risk patients [Diabetes Mellitus(T1DM), down syndrome (DS) and short status (SS)]

2. Evaluation of DGP-IgG and DGP-IgA in comparison with the other serological markers.

3. Evaluation of DQ2/ DQ8 typing for both diagnosis and epidemiology.

4. Evaluation of Small intestinal bacterial overgrowth (SIBO) among patients with celiac disease unresponsive to gluten-free diet (GFD).

These recommendations will be our plan for future separate publications.

\section{Ethical approval}

The collected data were part of a retrospective literature review; thus, a written ethical approval was not obtained before commencing the study.

\section{Disclosures}

Both authors have read and approved this manuscript. The current study was not funded or supported by any drug company. This paper is unique and is not under consideration by any other publication and has not been published elsewhere.

\section{Conflicts of interest}

The authors declare that they have no conflicts of interest.

\section{Author contributions}

Dr. Safi MA provided the framework of the study, wrote, reviewed and extensively edited the manuscript and gave it its final form. Both authors made the updated literature search, prepared the graphs and tables. Dr. Safi HMA also helped in the SPSS statistics.

\section{Financial support and sponsorship}

Nil.

\section{References}

1. Adams F (1956) The extant works of Aretaeus, the Cappodocian. Trans London: Sydenham Society.

2. Gee S (1988) On the coeliac disease. St Bart Hosp Rep 24: 17-20

3. Dicke WK, Weijers HA, Van De Kamer JH (1953) Coeliac disease. II. The presence in wheat of a factor having a deleterious effect in cases of coeliac disease. Acta Paediatr 42: 34-42. [Crossref]

4. Booth CC (1989) History of celiac disease. BMJ 298: 527-531. [Crossref]

5. Meuwisse GW (1970) Diagnostic criteria in coeliac disease. Acta Paediatr Scand 59: 461.

6. Visakorpi JK (1974) Definition of coeliac disease in children. In: Hekkens WThJM, Pena AS, eds. Coeliac disease. Proceedings of the Second International Coeliac Symposium, Noordwijkerhout, The Netherlands, Leiden: Stenfert Kroese, 1974: 10-16.

7. Seah PP, Fry L, Rossiter MA, Hoffbrand AV, Holborow EJ (1971) Anti-reticulin antibodies in childhood coeliac disease. Lancet 2: 681-682. [Crossref]

8. Bürgin-Wolff A, Hernandez R, Just M, Signer E (1976) Immunofluorescent antibodies against gliadin: a screening test for coeliac disease. Helv Paediatr Acta 31: 375-380. [Crossref]

9. O'Farrelly C, Kelly J, Hekkens W, Bradley B, Thompson A, et al. (1983) Alpha gliadin antibody levels: a serological test for coeliac disease. Br Med J (Clin Res Ed) 286: 2007-2010. [Crossref]

10. Chorzelski TP, Sulej J, Tchorzewska H, Jablonska S, Beutner EH, et al. (1983) IgA class endomysium antibodies in dermatitis herpetiformis and coeliac disease. Ann NY Acad Sci 420: 325-334. [Crossref]

11. Hill ID, Dirks MH, Liptak GS, Colletti RB, Fasano A, et al. (2005) Guideline for the diagnosis and treatment of celiac disease in children: recommendations of the North American Society for Pediatric Gastroenterology, Hepatology and Nutrition. J Pediatr Gastroenterol Nutr 40: 1-19. [Crossref]

12. [No authors listed] (1990) Revised criteria for diagnosis of coeliac disease. Report of Working Group of European Society of Paediatric Gastroenterology and Nutrition. Arch Dis Child 65: 909-911. [Crossref]

13. Guandalini S, Ventura A, Ansaldi N, Giunta AM, Greco L, et al. (1989) Diagnosis of coeliac disease: time for a change? Arch Dis Child 64: 1320-1324. [Crossref]

14. Marsh MN (1992) Gluten, major histocompatibility complex, and the small intestine. A molecular and immunobiologic approach to the spectrum of gluten sensitivity ('celiac sprue'). Gastroenterology 102: 330-354. [Crossref]

15. Oberhuber G, Granditsch G, Vogelsang H (1999) The histopathology of coeliac disease: time for a standardized report scheme for pathologists. Eur J Gastroenterol Hepatol 11: 1185-1194. [Crossref]

16. Dieterich W, Ehnis T, Bauer M, Donner P, Volta U, et al. (1997) Identification of tissue transglutaminase as the autoantigen of celiac disease. Nat Med 3: 797-801. [Crossref]

17. Mustalahti K, Catassi C, Reunanen A, Fabiani E, Heier M, et al. (2010) The prevalence of celiac disease in Europe: results of a centralized, international mass screening project. Ann Med 42: 587-595. [Crossref]

18. Rostom A, Murray JA, Kagnoff MF (2006) American Gastroenterological Association (AGA) Institute technical review on the diagnosis and management of celiac disease. Gastroenterology 131: 1981-2002. [Crossref]

19. Green PH, Cellier C (2007) Celiac disease. N Engl J Med 357: 1731-1743. [Crossref]

20. Barada K, Bitar A, Mokadem MA, Hashash JG, Green P (2010) Celiac disease in Middle Eastern and North African countries: a new burden? World J Gastroenterol 16: 1449-1457. [Crossref] 
21. Fouda MA (2017) Celiac disease-related osteopathy among Saudi celiac patients: Are we adherent to recommendations? Saudi J Gastroenterol 23: 291-295. [Crossref]

22. Saeed A, Assiri A, Assiri H, Ullah A, Rashid M (2017) Celiac disease in Saudi children. Evaluation of clinical features and diagnosis. Saudi Med J 38: 895-899. [Crossref]

23. Al-Hussaini A, Troncone R, Khormi M, AlTuraiki M, Alkhamis W, et al. (2017) Mass Screening for Celiac Disease Among School-aged Children: Toward Exploring Celiac Iceberg in Saudi Arabia. J Pediatr Gastroenterol Nutr 65: 646-651. [Crossref]

24. Fathy A, Abo-Haded HM, Al-Ahmadi N, El-Sonbaty MM (2016) Cardiac functions assessment in children with celiac disease and its correlation with the degree of mucosal injury: Doppler tissue imaging study. Saudi J Gastroenterol 22: 441-447. [Crossref]

25. Sarkhy A, El Mouzan MI, Saeed E, Alanazi A, Alghamdi S, et al. (2016) Socioeconomic Impacts of Gluten-Free Diet among Saudi Children with Celiac Disease. Pediatr Gastroenterol Hepatol Nutr 19: 162-167. [Crossref]

26. Al-Hakami AM1 (2016) Seroprevalence of coeliac disease in at-risk subjects at the main tertiary hospital, southwest of Saudi Arabia. Arab J Gastroenterol 17: 41-44. [Crossref]

27. Al-Hakami AM (2016) Pattern of thyroid, celiac, and anti-cyclic citrullinated peptide autoantibodies coexistence with type 1 diabetes mellitus in patients from Southwestern Saudi Arabia. Saudi Med J 37: 386-391. [Crossref]

28. Al-Mendalawi MD (2016) Prevalence of celiac disease in Saudi children: Is it underestimated? Saudi J Gastroenterol 22: 83. [Crossref]

29. Almadi MA, Aljebreen AM (2016) Celiac disease among symptom-free children-more than what is expected. Saudi J Gastroenterol 22: 82. [Crossref]

30. Al Hatlani MM (2015) Prevalence of celiac disease among symptom-free children from the Eastern Province of Saudi Arabia. Saudi J Gastroenterol 21: 367-71. [Crossref]

31. Saadah OI, Shaik NA, Banaganapalli B, Salama MA, Al-Harthi SE, et al. (2015) Replication of GWAS Coding SNPs Implicates MMEL1 as a Potential Susceptibility Locus among Saudi Arabian Celiac Disease Patients. Dis Markers 2015: 351673.

32. Sarkhy AA, El Mouzan MI, Saeed E, Alanazi A, Alghamdi S, et al. (2015) Clinical Characteristics of Celiac Disease and Dietary Adherence to Gluten-Free Diet among Saudi Children. Pediatr Gastroenterol Hepatol Nutr 18: 23-29. [Crossref]

33. Al-Agha AE, Alafif MM, Abd-Elhameed IA (2015) Glycemic control, complications, and associated autoimmune diseases in children and adolescents with type 1 diabetes in Jeddah, Saudi Arabia. Saudi Med J 36: 26-31. [Crossref]

34. Guandalini S, Assiri A (2014) Celiac disease: a review. JAMA Pediatr 168: 272-278. [Crossref]

35. Al-Hussaini A, Basheer A, Czaja AJ (2013) Liver failure unmasks celiac disease in a child. Ann Hepatol 12: 501-505. [Crossref]

36. Assiri A, Saeed A, AlSarkhy A, El Mouzan MI, El Matary W (2013) Celiac disease presenting as rickets in Saudi children. Ann Saudi Med 33: 49-51. [Crossref]

37. Al-Hussaini A, Sulaiman N, Al-Zahrani M, Alenizi A, El Haj I (2012) High prevalence of celiac disease among Saudi children with type 1 diabetes: a prospective crosssectional study. BMC Gastroenterol 12: 180. [Crossref]

38. Fouda MA, Khan AA, Sultan MS, Rios LP, McAssey K, et al. (2012) Evaluation and management of skeletal health in celiac disease: position statement. Can J Gastroenterol 26: 819-29. [Crossref]

39. Saadah OI, Al-Agha AE, Al Nahdi HM, Bokhary RY, Bin Talib YY, et al. (2012) Prevalence of celiac disease in children with type 1 diabetes mellitus screened by antitissue transglutaminase antibody from Western Saudi Arabia. Saudi Med J 33: 541-546. [Crossref]

40. Saadah OI, Al-Aama JY, Alaifan MA, Bin Talib YY, Al-Mughales JA (2012) Prevalence of celiac disease in children with Down syndrome screened by anti-tissue transglutaminase antibodies. Saudi Med J 33: 208-210.

41. Al-Jurayyan N NA, Mohamed SH, Al Otaibi HM, Al Issa ST, Omer HG (2012) Short stature in children: Pattern and frequency in a pediatric clinic, Riyadh, Saudi Arabia. Sudan J Paediatr 12: 79-83. [Crossref]

42. Al Jurayyan NA, Mohamed S, Al Issa SD, Al Jurayyan AN (2012) Rickets and osteomalacia in Saudi children and adolescents attending endocrine clinic, Riyadh, Saudi Arabia. Sudan J Paediatr 12: 56-63. [Crossref]

43. Saadah OI (2011) Celiac disease in children and adolescents at a singe center in Saudi Arabia. Ann Saudi Med 31: 51-57. [Crossref]

44. Bin-Abbas BS, Faiyaz-Ul-Haque M, Al-Fares AH, Al-Gazlan SS, Bhuiyan JA, et al. (2010) Autoimmune polyglandular syndrome type 1 in Saudi children. Saudi Med J 31: 788-792. [Crossref]
45. Assiri AM (2010) Isolated short stature as a presentation of celiac disease in Saudi children. Pediatr Rep 2: e4. [Crossref]

46. Ouda S, Saadah O, El Meligy O, Alaki S (2010) Genetic and dental study of patients with celiac disease. J Clin Pediatr Dent 35: 217-223. [Crossref]

47. Al-Ruhaily AD, Malabu UH (2009) Short stature in Saudi Arabia: etiologic profile in adult endocrine clinic. Niger J Med 18: 268-271. [Crossref]

48. Assiri AM, El Mouzan MI, Al Sanie A, Al Jurayyan N, Al Herbish AS, et al. (2008) Pattern of celiac disease in infants and children. Trop Gastroenterol 29: 217-220. [Crossref]

49. Al Hemidan AI, Tabbara KF, Althomali T (2006) Vogt-Koyanagi-Harada associated with diabetes mellitus and celiac disease in a 3-year-old girl. Eur J Ophthalmol 16 : 173-177. [Crossref]

50. Al Furaikh S, Al Zaben AA (2005) Recurrent small bowel intussusceptions: an uncommon presentation of celiac disease in an Arab child. Trop Gastroenterol 26: 3839. [Crossref]

51. Al-Ashwal AA, Shabib SM, Sakati NA, Attia NA (2003) Prevalence and characteristics of celiac disease in type I diabetes mellitus in Saudi Arabia. Saudi Med J24: 1113-1115. [Crossref]

52. Al-Mayouf SM, Al-Mehaidib AI, Alkaff MA (2003) The significance of elevated serologic markers of celiac disease in children with juvenile rheumatoid arthritis. Saudi J Gastroenterol 9: 75-78. [Crossref]

53. Fouda MA, Khan AA, Sultan M, Rios LP, McAssey K, et al. (2017) Corrigendum to "Evaluation and Management of Skeletal Health in Celiac Disease: Position Statement". Can J Gastroenterol Hepatol 2017: 1323607. [Crossref]

54. Al-Aama JY, Shaik NA, Banaganapalli B, Salama MA, Rashidi O, et al. (2017) Whole exome sequencing of a consanguineous family identifies the possible modifying effect of a globally rare AK5 allelic variant in celiac disease development among Saud patients. PLoS One 12: e0176664. [Crossref]

55. Tarabzouni S, AlKhairallah T (2017) Isolated Neurological Manifestation in Silent Celiac Disease. J Mov Disord 10: 105-107. [Crossref]

56. Banaganapalli B, Rashidi O, Saadah OI, Wang J, Khan IA, et al. (2017) Comprehensive Computational Analysis of GWAS Loci Identifies CCR2 as a Candidate Gene for Celiac Disease Pathogenesis. J Cell Biochem 118: 2193-2207. [Crossref]

57. Lihabi AA (2016) Trichotillomania in Celiac Disease. Case Rep Gastroenterol 10: 613 616. [Crossref]

58. Khan S, Mandal RK, Jawed A, Dar SA, et al. (2016) TNF-Ît -308 G > A (rs1800629) Polymorphism is Associated with Celiac Disease: A Meta-analysis of 11 Case-Control Studies. Sci Rep 6: 32677. [Crossref]

59. Al-Ajlan AS (2016) Screening of coeliac disease in undetected adults and patients diagnosed with irritable bowel syndrome in Riyadh, Saudi Arabia. Saudi J Biol Sci 23: 462-426. [Crossref]

60. Hasanato RM, Almomen AM (2015) Unusual presentation of arsenic poisoning in a case of celiac disease. Ann Saudi Med 35: 165-167. [Crossref]

61. Assiri AM, Saeed A, Saeed E, El-Mouzan MI, Alsarkhy AA, et al. (2015) Assessment of knowledge of celiac disease among health care professionals. Saudi Med J 36: 751753. [Crossref]

62. Al-Judaibi B, Driman DK, Chandok N (2015) An elderly lady with significant weight loss. Arab J Gastroenterol 16: 31-32. [Crossref]

63. Aljebreen AM, Almadi MA, Alhammad A, Al Faleh FZ (2013) Seroprevalence of celiac disease among healthy adolescents in Saudi Arabia. World J Gastroenterol 19: 2374-2378. [Crossref]

64. Khayyat YM (2012) Serologic markers of gluten sensitivity in a healthy population from the western region of Saudi Arabia. Saudi J Gastroenterol 18: 23-25. [Crossref]

65. Masoodi I, Wani H, Alsayari K, Sulaiman T, Hassan NS, et al. (2011) Celiac disease and autoimmune pancreatitis: an uncommon association. A case report. Eur J Gastroenterol Hepatol 23: 1270-1272. [Crossref]

66. Jammah AA (2012) A limp in a pregnant woman as a first presentation of celiac disease. Osteoporos Int 23: 1183-1186. [Crossref]

67. Alzahrani AS, Al Sheef M (2008) Severe primary hyperparathyroidism masked by asymptomatic celiac disease. Endocr Pract 14: 347-350. [Crossref]

68. Azzam NA, Al Ashgar H, Dababo M, Al Kahtani N, Shahid M (2006) Mesentric vein thrombosis as a presentation of subclinical celiac disease. Ann Saudi Med 26: 471-473. [Crossref] 
69. Al-Gahtani HA, Lingawi SS, Abdullah LS, Nooreddeen EA (2005) Osteomalacic myopathy due to celiac disease. Neurosciences (Riyadh) 10: 174-176. [Crossref]

70. Yasawy MI, Al-Quorain AA, Tamimi DM (2004) A typical adult celiac disease: report of cases and review of the literature. Saudi J Gastroenterol 10: 99-102. [Crossref]

71. Al Attas RA (2002) How common is celiac disease in Eastern Saudi Arabia? Ann Saudi Med 22: 315-319. [Crossref]

72. Qari FA (2002) Clinical presentation of adult celiac disease in Western Saudi Arabia. Saudi Med J 23: 1514-1517. [Crossref]

73. Al-Humaidi MA (2001) Adult celiac disease associated with type 1 diabetes. Saudi J Gastroenterol 7: 71-73. [Crossref]

74. Ahluwalia ML, Larbi EB, Fadali GA (1996) Adult celiac disease: Report of a case. Ann Saudi Med 16: 74-76. [Crossref]

75. Nazer HM, Abutalib H, Hugosson C, al-Mahr M, Ali MA (1991) Intestinal lymphangiectasia masquerading as coeliac disease. Ann Trop Paediatr 11: 349-355. [Crossref]

76. Vangone A, Abdel-Azeim S, Caputo I, Sblattero D, Di Niro R, et al. (2014) Structural basis for the recognition in an idiotype-anti-idiotype antibody complex related to celiac disease. PLoS One 9: e102839. [Crossref]

77. Selim ME, Al-Ayadhi LY (2013) Possible ameliorative effect of breastfeeding and the uptake of human colostrum against coeliac disease in autistic rats. World $J$ Gastroenterol 19: 3281-3290. [Crossref]

78. Alghamdi RA, Alghamdi AH, Fureeh AA (2018) Sero-Prevalence of Celiac Disease Among Symptom-Free Type 1 Diabetes Mellitus in Al-Baha Region, Saudi Arabia. IOSR J Pharm Biol Sci 13: 22-26.

79. Al-Qefari SN, Al-Twijri AW, Al-Adhadh AM, Al-Rashed OA, Al-Jarallah B (2018) Health-Related Quality of Life among Patients with Celiac Disease in Saudi Arabia. Ann Med Health Sci Res 8: 74-77.

80. Albrady A, Alharbi F, Almutairi A, Alhujaylan M, Almutiri N, et al. (2018) Clinical Profile of Celiac Disease: Patient Epidemiological Prospective. Ann Med Health Sci Res 8: 78-82.

81. Alharbi KL, Altwijri AW, Al-Shebromi AH, Alanazi IN, Alsweed MA, et al. (2017) Screening for Depressive Symptoms in Patients with Celiac Disease in Al-Qassim Region, Saudi Arabia. Ann Med Health Sci Res 7: 9-121

82. AlRuwaily F, Kattan HA, AlMehaidib AM, AlDekhail W (2017) Prevalence of celiac disease in Saudi children with Down syndrome: A retrospective study. Int J Pediatrics Adoles Med 4: 51-53.

83. Alshareef MA, Aljabri KS, Bokhari SA, Al Jiffri AM, Abu Elsaoud HM, et al. (2016) The Prevalence Of Celiac Disease in Saudi Patients with Type 1 Diabetes Mellitus: Cross Sectional Study. Int J Diabetes Metab Disord 1: 1-4.

84. Jouhari O (2013) Bone mineral densitometry in children using (DXA) scan-in Crohn's and celiac disease. In: 9th Applied Medical Sciences Students' Annual Meeting; King bdulaziz University, Dubai, 29.

85. AL Mehaidib A, Kattan H, AL Rrowaily F, AL Dekhail W (2011) Prevalence of celiac disease in Saudi children with Down syndrome: a retrospective study. J Gastroenterol Hepatol 26: 16-28.

86. Saadah OI, A Agha AE, Albokhari SM, A Mughales J (2004) A P0405 Screening of Saudi Children with Short Stature for Celiac Disease. J Pediat Gastroenterol Nut 39: S210.

87. Saadah OI, Al Agha AE, Albokhari SM, Al Mughales JA (2004) P0408 Disease in Saudi Childrine with type 1 Diabetes Mellitus. J Pediatr Gastroenterol Nut 39: S211.

88. Alahdal AM (2017) Misdiagnosed celiac disease: case report. Saudi J Intern Med 7: 43-46.

89. Ahmed M-EK, Nabrawi K, Al Shehri A, Siddiqui WA (2014) Enteropathy-Type T Cell Lymphoma Complicating Undiagnosed Celiac Disease in a Saudi Adult: Case Report. Am J Med Sci 4: 102-104.

90. Othman HI, Ouda SA (2010) Mandibular Radiomorphometric easurements as Indicators of Possible Osteoporosis in Celiac Patients. JKAU: Med Sci 17: 21-35.

91. Zawawi TH, Gangi MT, Hossain J, Mahgoub I, Sulieman R, et al. (1996) Adult celiac disease: Clinical features in patient seen in Western Region, Saudi Arabia. Saudi Med $J 17: 51-54$.

92. Al-Jurayyan N AM, Al Nemri AMH, Al Jurayyan ANA, Assiri AMA (2013) Celiac disease in children with short stature: A hospital-based study. J Taibah Univ Med Sci 8: 93-96.
93. Abdullah AMA (1990) Coeliac disease in Saudi-Arab children. Saudi Med J 11: 401404.] [Crossref]

94. Nazer H, Sakati N, Harfi HA (1990) Coeliac disease in Saudi Children. Proceedings of 4th annual paediatric symptoms. Ann Saudi Med 10: 232A.

95. Aktay AN, Lee PC, Kumar V, Parton E, Wyatt DT, et al. (2001) The prevalence and clinical characteristics of celiac disease in juvenile diabetes in Wisconsin. $J$ Pediatr Gastroenterol Nutr 33: 462-465. [Crossref]

96. Elfström P, Sundström J, Ludvigsson JF (2014) Systematic review with meta-analysis: associations between coeliac disease and type 1 diabetes. Aliment Pharmacol Ther 40: 1123-1132. [Crossref]

97. Hischenhuber C, Crevel R, Jarry B, Mäki M, Moneret-Vautrin DA, et al. (2006) Review article: safe amounts of gluten for patients with wheat allergy or coeliac disease. Aliment Pharmacol Therapeut 23: 559-575.

98. Megiorni F, Mora B, Bonamico M, Barbato M, Montuori M, et al. (2008) HLA-DQ and susceptibility to celiac disease: evidence for gender differences and parent-of-origin effects. Am J Gastroenterol 103: 997-1003. [Crossref]

99. Mustalahti K, Catassi C, Reunanen A, Fabiani E, Heier M, et al. (2010) The prevalence of celiac disease in Europe: results of a centralized, international mass screening project. Ann Med 42: 587-595. [Crossref]

100. Schuppan D, Junker Y, Barisani D (2009) Celiac disease: from pathogenesis to nove therapies. Gastroenterology 137: 1912-1933. [Crossref]

101. Abu-Zekry M, Kryszak D, Diab M, Catassi C, Fasano A (2008) Prevalence of celiac disease in Egyptian children disputes the east-west agriculture-dependent spread of the disease. J Pediatr Gastroenterol Nutr 47: 136-140. [Crossref]

102. Gujral N, Freeman HJ, Thomson AB (2012) Celiac disease: prevalence, diagnosis, pathogenesis and treatment. World Journal of Gastroenterology 18: 6036-6059. [Crossref]

103. Catassi C, Ratsch IM, Gandolfi L, Pratesi R, Fabiani E, et al. (1999) Why is coeliac disease endemic in the people of the Sahara? Lancet 354: 647-648. [Crossref]

104. Barker JM, Liu E (2008) "Celiac disease: pathophysiology, clinical manifestations, and associated autoimmune conditions". Adv Pediatr 55: 349-365. [Crossref]

105. Licks R, Licks V, Ourique F, Radke Bittencourt H, Fontanella V (2010) Development of a prediction tool for low bone mass based on clinical data and periapical radiography. Dentomaxillofac Radiol 39: 224-230. [Crossref]

106. McFarlane XA, Bhalla AK, Reeves DE, Morgan LM, Robertson DA (1995) Osteoporosis in treated adult coeliac disease. Gut 36: 710-714. [Crossref]

107. Lee BD, White SC (2005) Age and trabecular features of alveolar bone associated with osteoporosis. Oral Surg Oral Med Oral Pathol Oral Radiol Endod 100: 92-98. [Crossref]

108. Creo AL, Thacher TD, Pettifor JM, Strand MA, Fischer PR (2017) Nutritional rickets around the world: an update. Paediatr Int Child Health 37: 84-98. [Crossref]

109. [No authors listed] (2010) Rickets - OrthoInfo - AAOS.

110. Elidrissy AT (2016) The Return of Congenital Rickets, Are We Missing Occult Cases? Calcif Tissue Int 99: 227-236. [Crossref]

111. Sukriti K, Manish G, Syed RM, Abhinav GK, Keshav GK, et al. (2015) Prevalence and Clinical Profile of Celiac Disease in Patients with Type 1 Diabetes Mellitus in Western Uttar Pradesh, India. JAFES 30: 142-146.

112. Kaukinen K, Lindfors K, Collin P, Koskinen O, Mäki M (2010) Coeliac disease--a diagnostic and therapeutic challenge. Clin Chem Lab Med 48: 1205-1216. [Crossref]

113. Bhadada SK, Bhansali A, Kochhar R, Menon AS, Sinha SK, et al. (2015) Does Every Short Stature Child Need Screening for Celiac Disease? J Gastroenterol Hepatol 23: e353-e356. [Crossref]

114. Rossi TM, Albini CH, Kuma V (1993) Incidence of celiac disease identified by the presence of serum endomysial antibiodies in children with chronic diarrhea, short stature in Insulin Dependent Diabetes. J Pediatr 123: 262-264. [Crossref]

115. Lakhotia M, Pahadia HR, Kumar H, Singh J, Tak S (2015) A Case of Autoimmune Polyglandular Syndrome (APS) Type II with Hypothyroidism, Hypoadrenalism, and Celiac Disease - A Rare Combination. J Clin Diagn Res 9: OD01-OD03. [Crossref]

116. Hrubisková K, Jackuliak P, Vanuga P, Pura M, Payer J (2010) Autoimmune polyendocrine syndrome type 2 associated with autoimmune hypophysitis and coeliac disease. Vnitr Lek 56: 1169-1176. [Crossref] 
117. Stefano C, Priscilla L, Giuseppe F, Rosaria DG, Manuela P, et al. (2010) Prevalence of coeliac disease in rheumatoid and psoriatic arthritis and in psoriasis. Medit J Nut Metabol 3: 61-64.

118. Francis J, Carty JE, Scott BB (2002) The prevalence of coeliac disease in rheumatoid arthritis. Eur J Gastroenterol Hepatol 14: 1355-1356. [Crossref]

119. Lubrano E, Ciacci C, Ames PR, Mazzacca G, Oriente P, et al. (1996) The arthritis of coeliac disease: prevalence and pattern in 200 adult patients. Br J Rheumatol 35: 1314-1318. [Crossref]

120. García-Manzanares A, Lucendo AJ (2011) Nutritional and dietary aspects of celiac disease. Nutr Clin Pract 26: 163-173. [Crossref]

121. Leffler DA, Dennis M, Edwards-George JB, Jamma S, Magge S, et al. (2009) A simple validated gluten-free diet adherence survey for adults with celiac disease. Clin Gastroenterol Hepatol 7: 530-536. [Crossref]

122. Zanchi C, Ventura A, Martelossi S, Di Leo G, Di Toro N, et al. (2013) Rapid antitransglutaminase assay and patient interview for monitoring dietary compliance in celiac disease. Scand J Gastroenterol 48: 764-766. [Crossref]

123. Hall NJ, Rubin G, Charnock A (2009) Systematic review: adherence to a gluten-free diet in adult patients with coeliac disease. Aliment Pharmacol Ther 30: 315-330. [Crossref]

124. Ukkola A, Mäki M, Kurppa K, Collin P, Huhtala H, et al. (2012) Patients' experiences and perceptions of living with coeliac disease - implications for optimizing care. $J$ Gastrointestin Liver Dis 21: 17-22. [Crossref]

125. Schwertz E, Kahlenberg F, Sack U, Richter T, Stern M, et al. (2004) Serologic assay based on gliadin-related nonapeptides as a highly sensitive and specific diagnostic aid in celiac disease. Clin Chem 50: 2370-2375. [Crossref]

126. Barbato M, Maiella G, Di Camillo C, Guida S, Valitutti F, et al. (2011) The antideamidated gliadin peptide antibodies unmask celiac disease in small children with chronic diarrhoea. Dig Liver Dis 43: 465-469. [Crossref]
127. Mozo L, Gómez J, Escanlar E, Bousoño C, Gutiérrez C (2012) Diagnostic value of anti-deamidated gliadin peptide IgG antibodies for celiac disease in children and IgAdeficient patients. J Pediatr Gastroenterol Nutr 55: 50-55. [Crossref]

128. Neves MM, Gonzalez-Garcia MB, Nouws HP, Costa-Garcia A (2013) An electrochemical deamidated gliadin antibody immunosensor for celiac disease clinical diagnosis. Analyst 138: 1956-1958. [Crossref]

129. Leffler DA, Schuppan D (2010) Update on serologic testing in celiac disease. Am J Gastroenterol 105: 2520-2524. [Crossref]

130. Nandiwada SL, Tebo AE (2013) Testing for antireticulin antibodies in patients with celiac disease is obsolete: a review of recommendations for serologic screening and the literature. Clin Vaccine Immunol 20: 447-451. [Crossref]

131. Parizade M, Bujanover Y, Weiss B, Nachmias V, Shainberg B (2009) Performance of serology assays for diagnosing celiac disease in a clinical setting. Clin Vaccine Immunol 16: 1576-1582. [Crossref]

132. Megiorni F, Pizzuti A (2012) HLA-DQA1 and HLA-DQB1 in Celiac disease predisposition: practical implications of the HLA molecular typing. J Biomed Sci 19: 88. [Crossref]

133. Cecilio LA, Bonatto MW2 (2015) The prevalence of HLA DQ2 and DQ8 in patients with celiac disease, in family and in general population. Arq Bras Cir Dig 28: 183185. [Crossref]

134. Tursi A, Brandimarte G, Giorgetti G (2003) High prevalence of small intestina bacterial overgrowth in celiac patients with persistence of gastrointestinal symptoms after gluten withdrawal. Am J Gastroenterol 98: 839-843. [Crossref]

135. Ghoshal UC, Ghoshal U, Misra A, Choudhuri G (2004) Partially responsive celiac disease resulting from small intestinal bacterial overgrowth and lactose intolerance. BMC Gastroenterol 4: 10. [Crossref]

Copyright: (C2018 Safi MA. This is an open-access article distributed under the terms of the Creative Commons Attribution License, which permits unrestricted use, distribution, and reproduction in any medium, provided the original author and source are credited. 